\title{
Quenching or Bursting: Star Formation Acceleration-A New Methodology for Tracing Galaxy Evolution
}

\author{
D. Christopher Martin ${ }^{1}$, Thiago S. Gonçalves ${ }^{2}$, Behnam Darvish ${ }^{1}$, Mark Seibert ${ }^{1}$, and David Schiminovich ${ }^{3}$ \\ ${ }^{1}$ California Institute of Technology, MC 405-47, 1200 East California Boulevard, Pasadena, CA 91125, USA \\ ${ }^{2}$ Observatorio do Valongo, Universidade Federal do Rio de Janeiro, Ladeira Pedro Antonio, 43, Saude, Rio de Janeiro-RJ 20080-090, Brazil \\ ${ }^{3}$ Department of Astronomy, Columbia University, New York, NY 10027, USA \\ ${ }^{4}$ Observatories of the Carnegie Institution of Washington, 813 Santa Barbara St., Pasadena, CA 91101, USA \\ Received 2016 October 8; revised 2017 April 10; accepted 2017 May 5; published 2017 June 8
}

\begin{abstract}
We introduce a new methodology for the direct extraction of galaxy physical parameters from multiwavelength photometry and spectroscopy. We use semianalytic models that describe galaxy evolution in the context of largescale cosmological simulation to provide a catalog of galaxies, star formation histories, and physical parameters. We then apply models of stellar population synthesis and a simple extinction model to calculate the observable broadband fluxes and spectral indices for these galaxies. We use a linear regression analysis to relate physical parameters to observed colors and spectral indices. The result is a set of coefficients that can be used to translate observed colors and indices into stellar mass, star formation rate, and many other parameters, including the instantaneous time derivative of the star formation rate, which we denote the Star Formation Acceleration (SFA), We apply the method to a test sample of galaxies with GALEX photometry and SDSS spectroscopy, deriving relationships between stellar mass, specific star formation rate, and SFA. We find evidence for a mass-dependent SFA in the green valley, with low-mass galaxies showing greater quenching and higher-mass galaxies greater bursting. We also find evidence for an increase in average quenching in galaxies hosting an active galactic nucleus. A simple scenario in which lower-mass galaxies accrete and become satellite galaxies, having their star-forming gas tidally and/or ram-pressure stripped, while higher-mass galaxies receive this gas and react with new star formation, can qualitatively explain our results.
\end{abstract}

Key words: galaxies: evolution - galaxies: star formation - ultraviolet: galaxies

\section{Introduction}

There has been growing interest in the nature of the observed color bimodality in the distribution of galaxies (Baldry et al. 2004; Balogh et al. 2004), which is echoed in other galaxy properties (Kauffman et al. 2003). The color bimodality is revealed in a variety of color-magnitude plots, and is particularly dramatic in the UV-optical color-magnitude diagram (Wyder et al. 2007). The red and blue galaxy concentrations are commonly denoted the red sequence and the blue "cloud," although we elect to call both concentrations sequences. Recently the blue cloud translated into the specific star formation rate (sSFR)-stellar mass plane has become tight enough to be denoted a blue sequence or a "main sequence" for star-forming galaxies. Deep galaxy surveys are now probing the evolution of the red and blue sequences. Work using the COMBO-17 (Bell et al. 2004), DEEP2 (Willmer et al. 2006; Faber et al. 2007), and more recently the UltraVISTA (Ilbert et al. 2013) surveys provides evidence that the red sequence has grown in mass by a factor of three since $z \sim 1$. It is natural to ask what processes have led to this growth, and in particular whether the red sequence has grown via gas-rich mergers, gasless (dry) mergers, or simple gas exhaustion. There is also considerable controversy regarding whether feedback from an active galactic nucleus (AGN) has played a role in accelerating this evolution, with many authors supporting this hypothesis (e.g., Di Matteo et al. 2005; Springel et al. 2005; Maiolino et al. 2012; Dubois et al. 2013; Olsen et al. 2013; Shimizu et al. 2015), and many others claiming that energy injection from other feedback mechanisms would dominate the quenching process (e.g., Coil et al. 2011; Aird et al. 2012).
Likewise, it has long been assumed that environment plays a significant role in quenching star formation in galaxies. In his seminal work, Dressler (1980) has shown a strong relation between galaxy morphology and the local density, a relation that translates to an environmental dependence of color and star formation properties on environment (e.g., Balogh et al. 2004; Blanton et al. 2005; Zehavi et al. 2005; Darvish et al. 2016). Peng et al. (2010) have shown that this dependence is stronger for low-mass galaxies, indicating that quenching of satellite galaxies in clusters is particularly relevant. Peng et al. (2015) have later argued that the main quenching mechanism in galaxies is "strangulation" within clusters. Nevertheless, this result relies on average metallicities and star formation properties of tens of thousands of galaxies, without any regard to processes happening within individual galaxies.

Therefore, we would like very much to identify galaxies that may be in the process of evolving from the blue to the red sequence. Martin et al. (2007, henceforth M07) have made a first attempt using the $D_{n}(4000)$ and $H \delta_{A}$ indices as defined in Kauffman et al. (2003), and inferred the total mass flux between the two sequences at redshift $z \sim 0.1$. Gonçalves et al. (2012) have extended the analysis to intermediate redshifts $(z \sim 0.8)$ and noticed an increased mass flux density at earlier times and for more massive galaxies, meaning that the phenomenon of star formation quenching has suffered a sizeable reduction in the last 6-7 Gyr. Nevertheless, these results rely on the (simplistic) assumption of a star formation history dominated by an exponential decrease in star formation rates in all green valley galaxies, which cannot be true.

In this paper, we develop a new methodology inspired by earlier work developing simple broadband and spectral index 
fitting formulae (Calzetti et al. 2000; Kauffman et al. 2003; Seibert et al. 2005; Johnson et al. 2007a, 2007b) designed to extract physical parameters without explicit fitting of the spectral energy distribution (SED). Our method starts with model galaxies produced by a semianalytic model set based on an $N$-body cosmological simulation (Millennium) (Section 2). We then use a linear regression technique to relate photometric and spectral index observables for models binned by the $D_{n}(4000)$ spectral index to model galaxy physical parameters and star formation histories (Section 3). We define a new star formation history parameter the Star Formation Acceleration (SFA), which is the time derivative of the NUV $-i$ color (Section 3.2). A positive SFA corresponds to a galaxy that is quenching (SFR and sSFR are dropping), while a negative SFA indicates a bursting change in SFR (SFR and SSFR are increasing). We apply this to a matched test sample of SDSSGALEX galaxies and derive some interesting preliminary results (Section 4).

Throughout this work, we assume a flat $\Lambda$ CDM cosmology with $H_{0}=70 \mathrm{~km} \mathrm{~s}^{-1} \mathrm{Mpc}^{-1}, \Omega_{m}=0.3$, and $\Omega_{\Lambda}=0.7$. Magnitudes are expressed in the $\mathrm{AB}$ system (Oke \& Gunn 1983) and stellar mass and star formation rates are based on a Salpeter initial mass function (Salpeter 1955).

\section{Method: Galaxy Models}

One of the principal activities in the field of galaxy evolution is the translation of multiwavelength photometry and spectroscopy into galaxy physical parameters. The vast majority of methods use a SED fitting approach. Modelers translate physical parameters and star formation histories into SEDs, and search for the SED (and corresponding parameters) or range of SEDs that give the best statistical fit. Examples of such an approach are given by Kauffman et al. (2003) and Salim et al. (2005, 2007), who use a Bayesian analysis of observations fit to a large library of model SEDs that populate galaxy physical parameter space. The outputs include probability distributions for derived physical parameters.

At the same time, a number of workers have shown that in certain cases simple fitting formulae can provide a direct translation of observables into physical parameters. For example, the UV slope is related to the infrared excess (IRX, the ratio of far or total infrared luminosity to far UV luminosity) for starburst (Calzetti et al. 2000) and normal (Seibert et al. 2005) galaxies. More complex fitting formulae can be derived using the $D_{n}(4000)$ spectral index (Johnson et al. 2007a, 2007b). Kauffman et al. (2003) and these papers demonstrated that $D_{n}(4000)$ does an excellent job of isolating the age of a stellar population from other parameters such as extinction.

This paper introduces a generalization of the fitting formula approach to many physical parameters and moments of the star formation history. A summary of the approach follows:

1. We use a semianalytic model (De Lucia 2006) linked to the Millennium cosmological $N$-body simulation (Springel et al. 2005) to provide a large sample of galaxies, star formation histories, and associated physical parameters.

2. We use a simple extinction model and stellar population synthesis code to translate the star formation histories into observable broadband fluxes and spectral indices.
3. We bin model SEDs by $D_{n}(4000)$ to remove the principal source of variation, stellar population age.

4. Within each $D_{n}(4000)$ bin we perform a linear regression fit between model physical parameters and the multiple observables (colors and spectral indices) for the complete galaxy sample. We find in general linear (in the $\log$ ) relationships between the two over a large dynamic range. Fit dispersion varies with physical parameter and with the collection of available observables.

5. The matrix of regression coefficients can be used to translate observables into physical parameters (after introducing some offsets), and to derive observable influence functions, degeneracies, and error propagation matrices.

\subsection{Cosmological Simulation and Semianalytic Model}

We use a set of 24,000 model galaxies produced by the semianalytic model (SAM) of De Lucia (2006) applied to the Millennium cosmological simulation. Galaxies are modeled in 63 time steps of $\sim 300 \mathrm{Myr}$ each over the redshift range $0<z<6$.

The Millennium simulation (Springel et al. 2005) is an $N$-body simulation that follows $2160^{3}$ particles since redshift $z=127$ in a cosmological volume $500 h^{-1} \mathrm{Mpc}$ on a side. Assuming a cold dark matter cosmology, it provides a framework in which one can follow the formation of dark matter halos and the large-scale structure on cosmologically significant scales. De Lucia (2006) used this framework and applied a semianalytic model which, following dark matter halos even after accretion onto larger systems, assumed a star formation law that depended on the mass of cold gas and a minimum critical value of gas surface density above which new stars were allowed to form. With the addition of feedback from AGNs, the authors are able to reproduce the observed trend of short formation timescales of the most massive elliptical galaxies (e.g., Thomas et al. 2005).

We used 24,000 galaxies (at snapnum $=63$ or $z=0$ ) from the volume range $(0<x<65 \mathrm{Mpc}, 0<y<65 \mathrm{Mpc}, 0<$ $z<65 \mathrm{Mpc}$ ), where $x, y$, and $z$ are the galaxy coordinates in the Millennium catalog, and absolute magnitude $M_{r}<-17$. Each $z=0$ galaxy is the base of a merger tree. Each tree and all galaxy predecessors were loaded, giving a total of 900,000 galaxy models over all 63 time steps and over the redshift range $0<z<6$. All regression fits given below use all galaxies in all time steps (subdivided only by $D_{n}(4000)$ and in nine coarse redshift bins), using rest-frame observables. Hence, all results given below can be applied to galaxies at any redshift, using $k$-corrected observables.

\subsection{Spectral Energy Distributions}

\subsubsection{Stellar Population Synthesis}

We use the SAM model star formation rate for each galaxy and the merger tree to calculate a star formation history for each galaxy at each time step/redshift. The star formation rate (SFR) versus time is calculated at each time step and is the sum of the star formation histories of all predecessor galaxies in the merger tree. Updated models of stellar population synthesis of Bruzual \& Charlot (2003) using a single stellar population (SSP) are used to predict broadband luminosities and spectral 

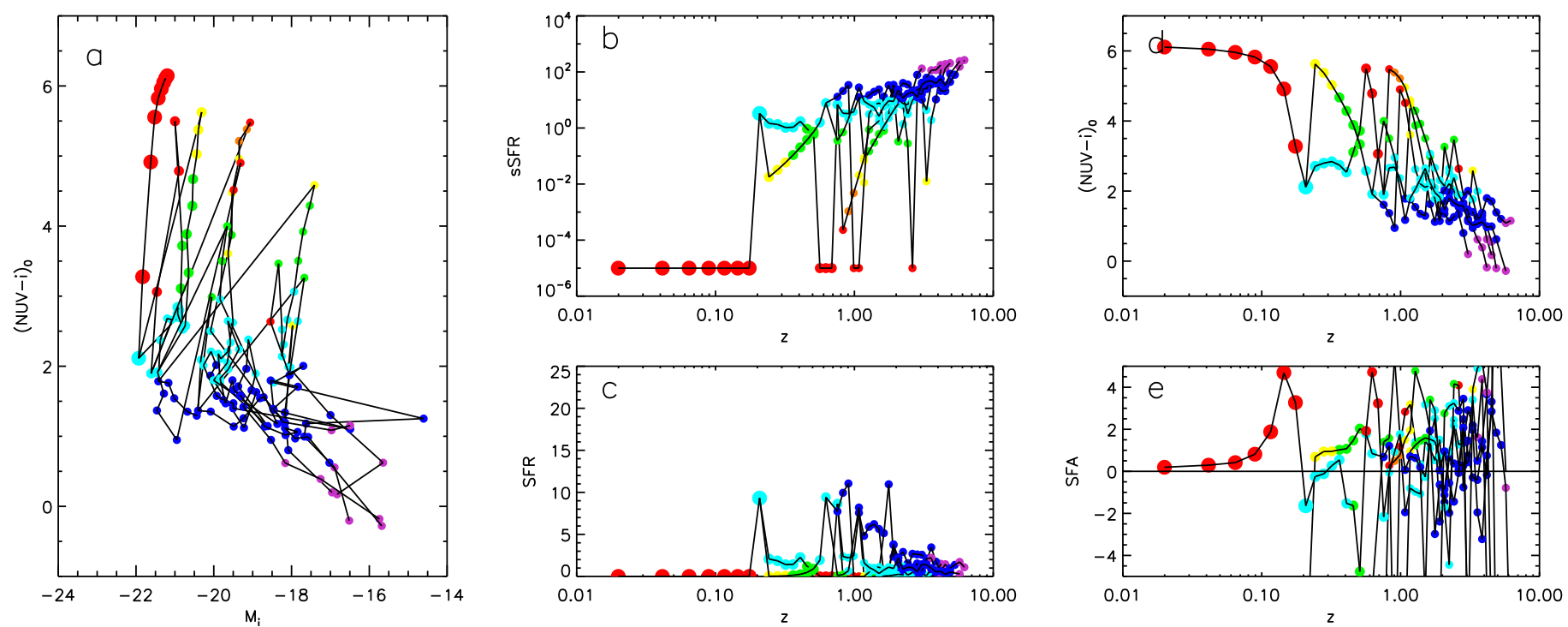

Figure 1. Simulated star formation history for a galaxy most like a massive quiescent at the present day. (a) Color-magnitude diagram (extinction-corrected NUV $-i$ vs. $M_{i}$ ) showing evolutionary tracks of all the galaxies that eventually merge into the single quiescent galaxy. The final merger occurs at $z=0.2$. Circle size is keyed to $\log \left(M_{*}\right)$, and color is keyed to specific star formation rate. (b) Specific SFR (sSFR) vs. redshift for all constituent and final galaxies. (c) SFR vs. redshift plotted as in panel (b). (d) NUV - $i$ vs. redshift. (e) Star formation acceleration (see Section 3.2) vs. redshift.
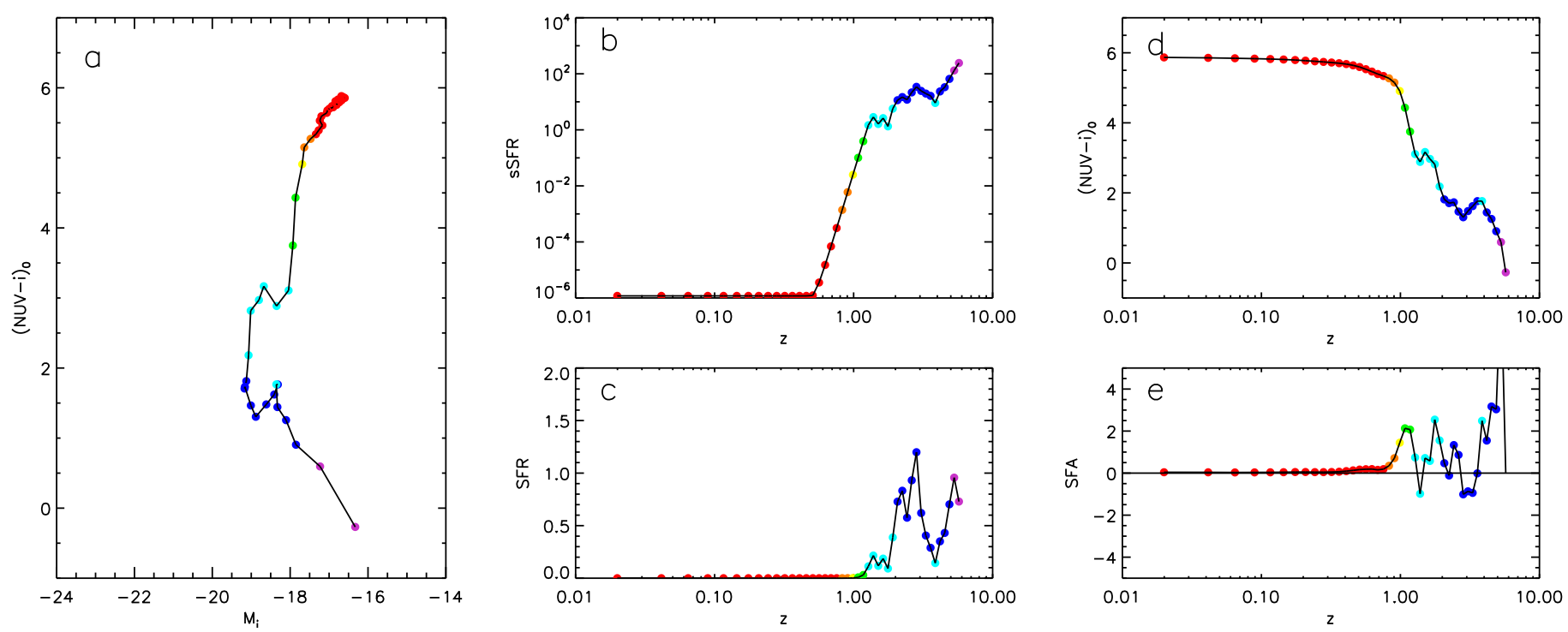

Figure 2. Simulated star formation history for a galaxy starting a slow quench at $z \sim 2$. (a) Color-magnitude diagram (extinction-corrected NUV $-i$ vs. $\left.M_{i}\right)$. Circle size is keyed to $\log \left(M_{*}\right)$, and color is keyed to specific star formation rate. (b) Specific SFR (sSFR) vs. redshift for all constituent and final galaxies. (c) SFR vs. redshift plotted as in panel (b). (d) NUV - $i$ vs. redshift. (e) Star formation acceleration (see Section 3.2) vs. redshift.

indices. These models are available in seven metallicity bins. In each time step, an SSP is created associated with the SFR and time interval in that time step. The metallicity of the SSP in this time step is derived from the gas-phase metallicity from the SAM (using the closest available SSP model). We use a Salpeter initial mass function (IMF).

\subsubsection{Dust Extinction}

We have used a simple geometric model for dust extinction. The SAM predicts gas-phase metallicity $\left(Z_{\text {gas }}\right)$, gas mass $\left(M_{\mathrm{gas}}\right)$, and galaxy size $\left(r_{\mathrm{gal}}\right)$. We assume that gas and dust are distributed in a uniform absorbing slab with selective extinction $E_{B-V}$ given by

$$
E_{B-V}=C_{0} \mu Z_{\mathrm{gas}} M_{\mathrm{gas}} r_{\mathrm{gal}}^{-2}(\cos i)^{-1}
$$

where $C_{0}$ is a constant (obtained by using the Milky Way values) and $i$ is the galaxy inclination. The constant $\mu$ allows for a larger absorption for young stars than for evolved stars (Calzetti et al. 1994). We use $\mu=1$ for stars younger than $10 \mathrm{Myr}$ and $\mu=0.5$ for older stars.

We use three possible extinction-law models. (1) The starburst extinction law from Calzetti et al. (2000) gives the 

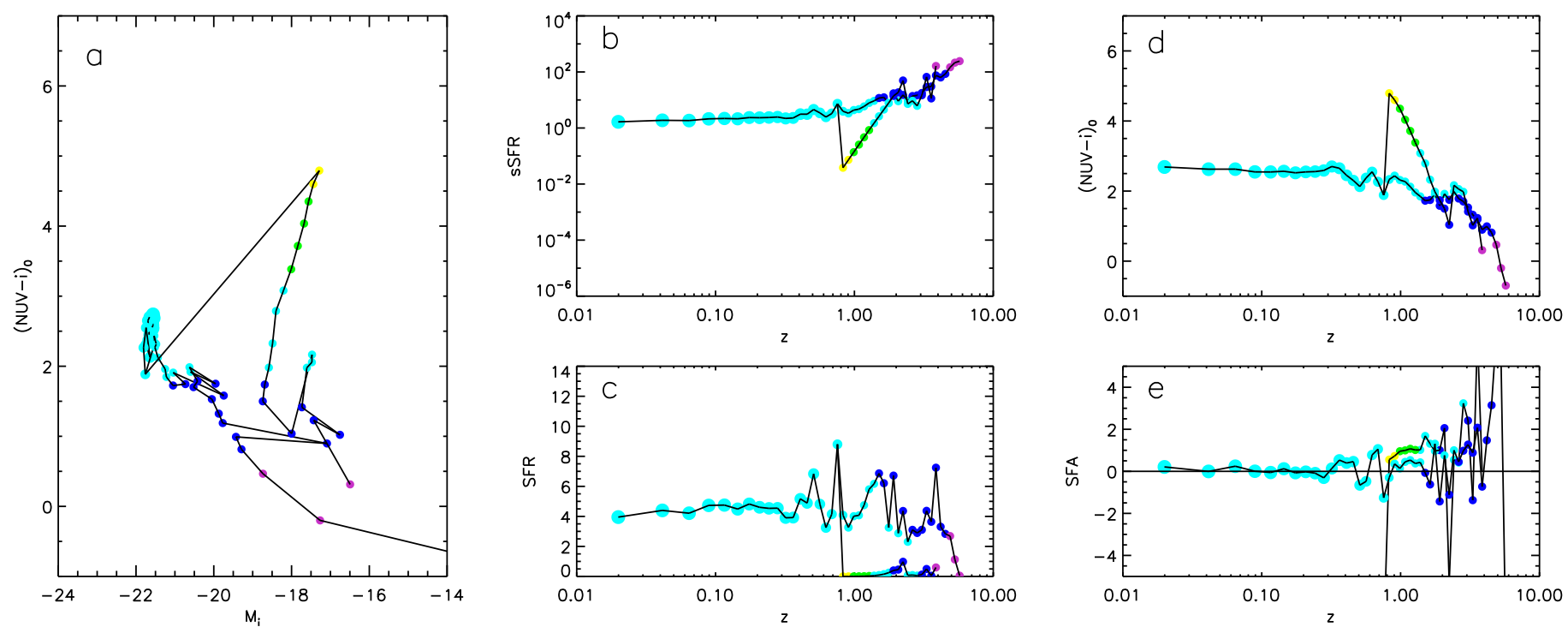

Figure 3. Simulated star formation history for a galaxy like a star-forming one with a late minor merger at $z \sim 0.8$. (a) Color-magnitude diagram (extinction-corrected NUV $-i$ vs. $M_{i}$ ) showing evolutionary tracks of all the galaxies that eventually merge into the single galaxy. Circle size is keyed to $\log \left(M_{*}\right)$, and color is keyed to specific star formation rate. (b) Specific SFR (sSFR) vs. redshift for all constituent and final galaxies. (c) SFR vs. redshift plotted as in panel (b). (d) NUV - $i$ vs. redshift. (e) Star formation acceleration (see Section 3.2) vs. redshift.
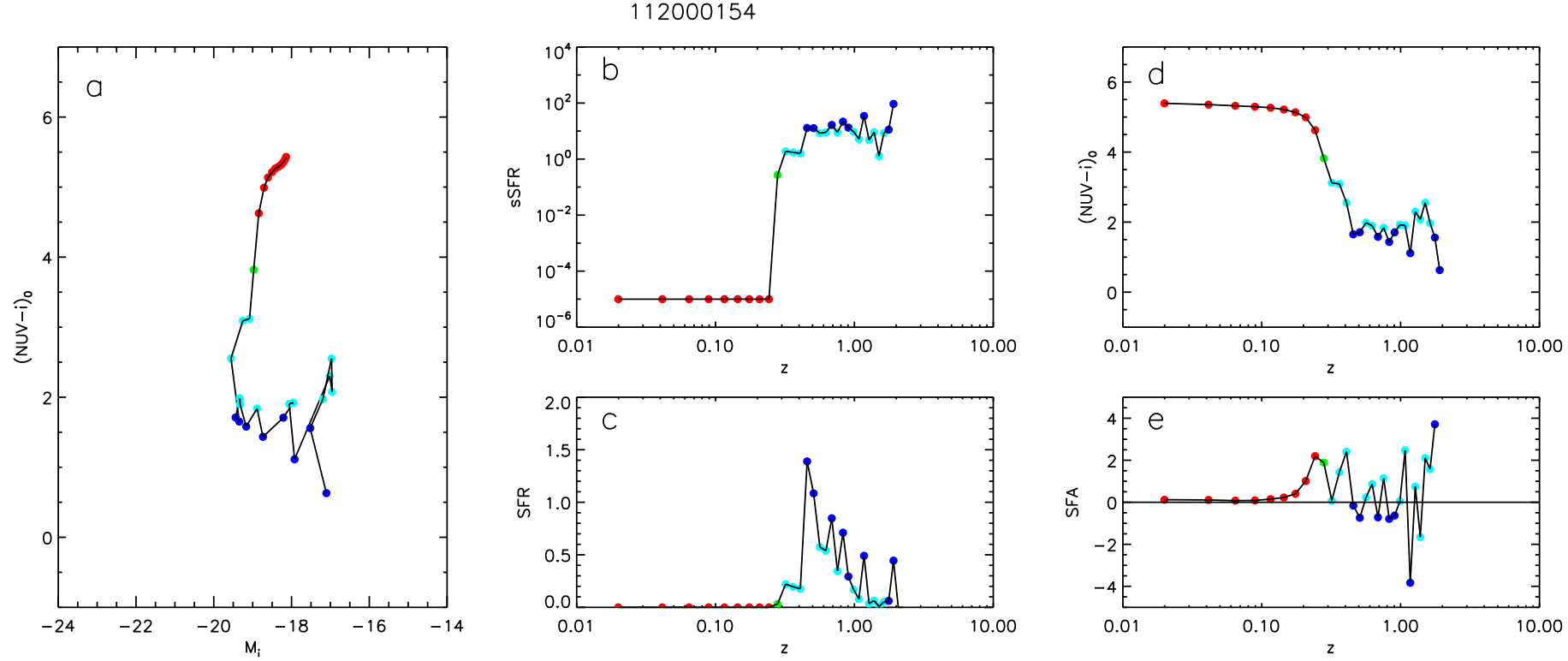

Figure 4. Simulated star formation history for a galaxy starting a fast quench at $z \sim 0.5$. (a) Color-magnitude diagram (extinction-corrected NUV $-i$ vs. $M_{i}$ ) showing evolutionary tracks for the galaxy. Circle size is keyed to $\log \left(M_{*}\right)$, and color is keyed to specific star formation rate. (b) Specific SFR (sSFR) vs. redshift for all constituent and final galaxies. (c) SFR vs. redshift plotted as in panel (b). (d) NUV - $i$ vs. redshift. (e) Star formation acceleration (see Section 3.2) vs. redshift.

usual $A_{\mathrm{FUV}}$ or IRX versus UV slope with $A_{\mathrm{FUV}}$ and IRX increasing with $\beta$, the slope of the SED in the FUV/NUV region. (2) The Milky Way extinction law from Cardelli et al. (1989) has an IRX- $\beta$ relationship that is flattened and even reversed because of the $2200 \AA$ bump. (3) A mixed extinction model in which a fraction $f_{M}$ of the dust follows Milky Way extinction, and a fraction $1-f_{M}$ follows the starburst extinction, where $f_{M}$ is chosen randomly over a range $0<f_{M}<f_{M \text {,max }}$. For the results given below we use this third method, which gives a fitting error of $0.3 \mathrm{mag}$ for $f_{M, \max }=0.5$, rising to $0.5 \mathrm{mag}$ for $f_{M, \max }=1$. In order to incorporate the positive definite quantity $A_{\mathrm{FUV}}$ as a derived parameter, we fit the quantity $\mathrm{IRX}_{\mathrm{FUV}}=\log _{10}\left(10^{0.4 A_{\mathrm{FUV}}}-1\right)$.

\subsubsection{Nebular Emission}

We do not incorporate nebular emission in this version of the model. In a future paper we will incorporate emission lines and examine additional physical parameters that these trace, including SFR and IMF. 

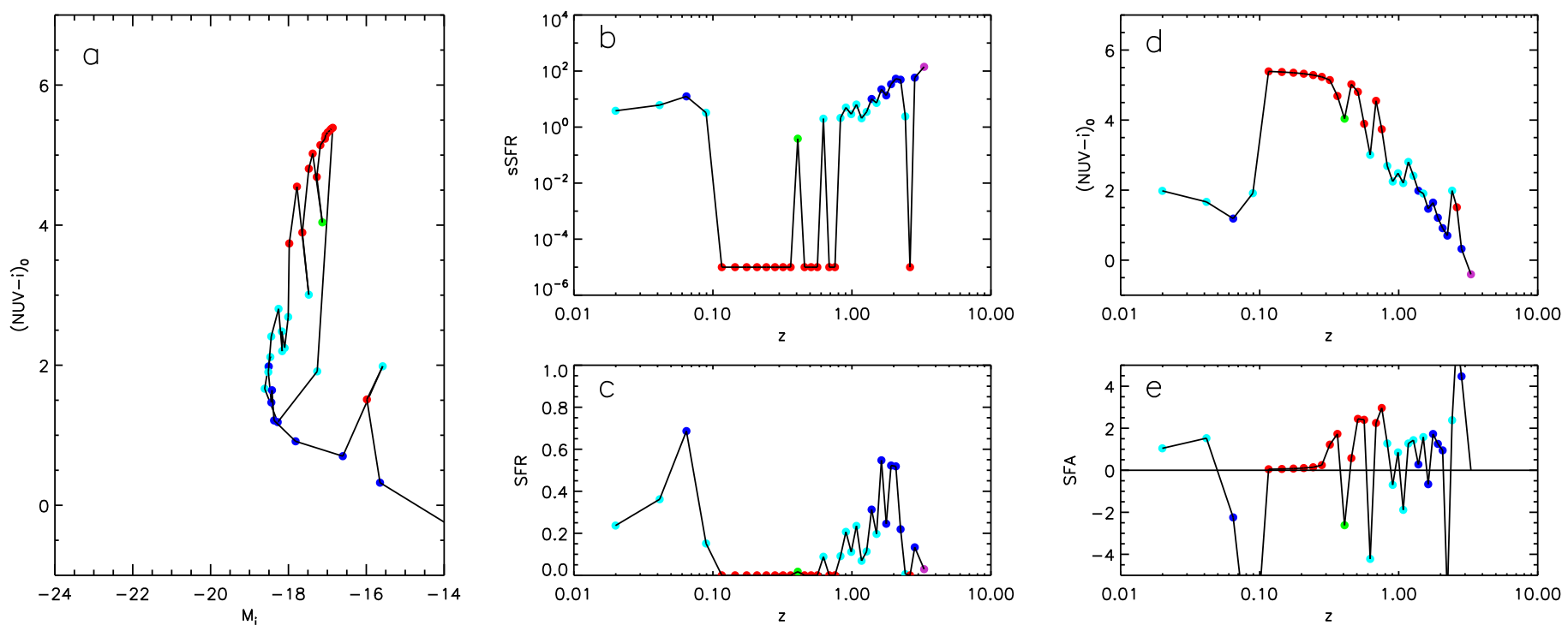

Figure 5. Simulated star formation history for a dwarf galaxy quenching at early times and then bursting at $z \sim 0.07$. (a) Color-magnitude diagram (NUV $-i$ vs. $M_{i}$ including extinction) showing evolutionary tracks in the galaxy. Circle size is keyed to $\log \left(M_{*}\right.$ ), and color is keyed to specific star formation rate. (b) Specific SFR (sSFR) vs. redshift for all constituent and final galaxies. (c) SFR vs. redshift plotted as in panel (b). (d) NUV - $i$ vs. redshift. (e) Star formation acceleration (see Section 3.2) vs. redshift.

\subsubsection{Sample Star Formation Histories}

In Figures 1-5 we show sample star formation histories and evolution in the NUV $-i$ versus $M_{i}$ color-magnitude diagram from five galaxies (a massive quiescent, a galaxy slowquenching at $z \sim 2$, a disk galaxy with relatively constant SFR, a galaxy fast-quenching at $z \sim 0.5$, and a low-mass recent starburst galaxy).

\section{Method: Galaxy Physical Parameters}

\subsection{Mathematical Motivation}

We would like to recover measures of recent star formation history that are nonparametric. Our technique relies on linearization, effectively Taylor expansion to the linear term of a multidimensional nonlinear function around fixed points. There is a complex, nonlinear relationship between observed colors and spectral indices and physical parameters. For an SSP the principal source of variation is age. A robust measure of SSP age is the spectral index $D_{n}(4000)$, since extinction has almost no effect (metallicity has some effect, and we defer discussion of this until Section 5). We make an ansatz that once $D_{n}(4000)$ is specified, there is a linear relationship between observable colors and indices and star formation metrics such as SFR, specific SFR, stellar mass, and recent changes in SFR. This relationship can be tested with a family of star formation histories and a model of stellar population synthesis, as long as this family spans the space of real galaxy star formation histories. We also assume that physical parameters such as stellar and gas metallicity, gas mass, and extinction also have this linear relationship with observables. Testing this requires relating the star formation histories to the physical parameters with, for example, a semianalytic model connected to a realistic cosmological simulation.

\subsection{Regression Method and SFA Parameter}

We use standard multiple linear regression (MLR) to relate physical parameters to observed properties. For this initial work, we use the following observables. All samples are binned in $D_{n}(4000)$ with $\Delta D_{n}(4000)=0.05$. Other observables used in this initial study are the colors: FUV - NUV, NUV $-u, u-g, g-r, r-i$; the spectral index $H \delta_{A}$, and the absolute magnitude $M_{i}$. FUV and NUV are GALEX bands, and $u, g, r, i, z$ are SDSS bands.

We perform MLR between all of these observables and each of the following physical parameters: stellar mass $\left(\log M_{*}\right)$, star formation rate $(\operatorname{logSFR})$, FUV extinction $\left(A_{\mathrm{FUV}}\right)$, extinction correction to NUV $-i\left(\Delta(\mathrm{NUV}-i)=(\mathrm{NUV}-i)_{0}\right.$ $-(\mathrm{NUV}-i)_{\mathrm{obs}}$ where (NUV $\left.-i\right)_{0}$ is the extinction-corrected NUV - $i)$, mass-weighted stellar age $\left(\log t_{*}\right)$, gas mass $\left(\log M_{\mathrm{gas}}\right)$, gas metallicity $\left(Z_{\mathrm{gas}}\right)$, and stellar metallicity $\left(Z_{*}\right)$.

We also fit two additional functions related to moments of the star formation history. We call the "star formation acceleration" the time derivative of the extinction-corrected $\mathrm{NUV}-i$ color $\left(\mathrm{SFA} \equiv d(\mathrm{NUV}-i)_{0} / d t\right)$ (note that the SFA defined using NUV $-r$ and that defined using NUV $-i$ differ by only $1 \%$ ). The SFA is calculated using the current and previous time steps, and is quantified in the units mag $\mathrm{Gyr}^{-1}$. In the lowest redshift bin $(0<z<0.3)$, applicable in the results we present below, the time steps are separated by $0.3 \mathrm{Gyr}$.

While there are several possible definitions one could use for SFA (specifically, $d$ (SFR) $/ d t, d(\mathrm{sSFR}) / d t, d \log (\mathrm{sSFR}) / d t$, and $d$ (NUV $-i)_{0} / d t$ ), we have chosen to use the last of these for the following reasons. (1) $d$ (SFR) $/ d t$ is not massnormalized and will scale with galaxy mass, making direct comparisons between mass bins less informative. (2) sSFR can vary over many orders of magnitude, making comparisons of galaxies in different sSFR bins less informative. (3) $d \log (\mathrm{sSFR}) / d t$ is more useful and can track changes across the color-magnitude diagram (CMD). But it can take on large 

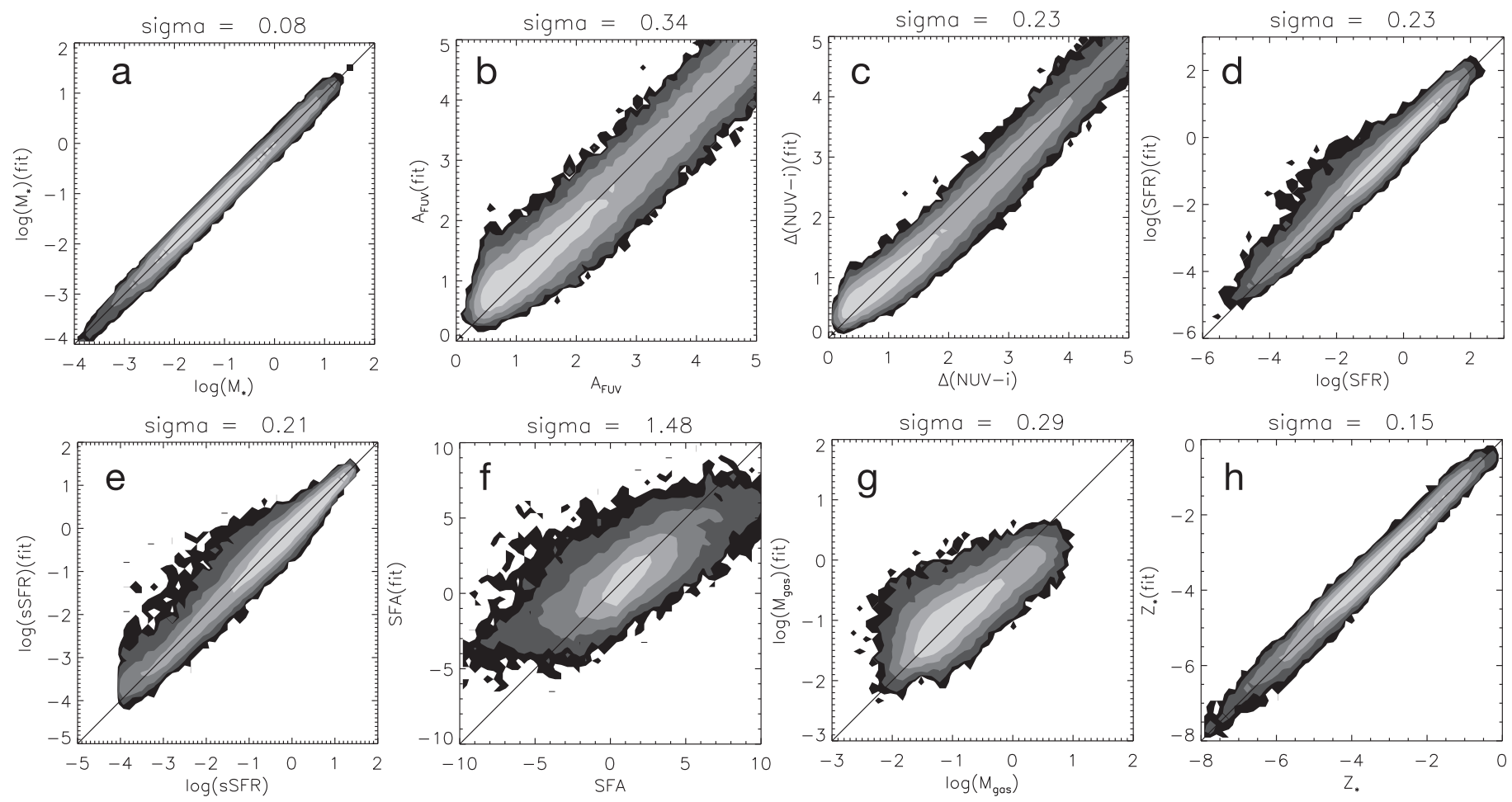

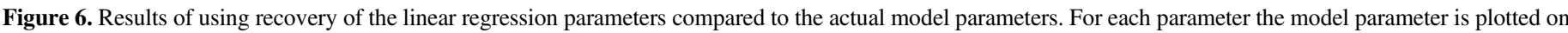

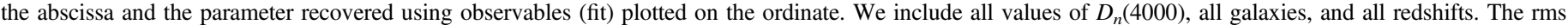

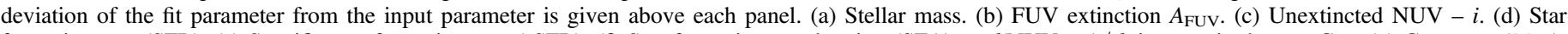

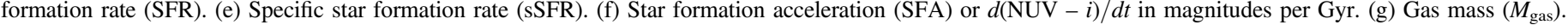
(h) Stellar $\left(Z_{*}\right)$.

negative and even indefinite values when quenching occurs rapidly that can only be bounded by using arbitrary parameters to limit the change. We have experimented with using log (sSFR), finding that the fits are significantly worse than with our adopted definition $\left(2.5 \sigma\left(\mathrm{SFA}_{\mathrm{sSFR}}\right)=4.4\right.$ versus $\left.\sigma\left(\mathrm{SFA}_{\mathrm{NUV}-i}\right)=1.5\right)$. (4) Our adopted definition is logarithmic and $(\mathrm{NUV}-i)_{0}$ is well correlated with $\log (\mathrm{sSFR})$ (with $(\mathrm{NUV}-i)_{0} \simeq$ const. $-2.5 \log (\mathrm{sSFR})$ for $1<(\mathrm{NUV}-i)_{0}<$ 5 with a break and a slightly shallower function for $(\mathrm{NUV}-i)_{0}>5$ ). It is also well behaved even with abrupt changes in SFR and sSFR. Because it and all the observed colors and spectral indices are light-weighted moments of the star formation history they are better correlated and the fit dispersions much lower. Finally, using this definition we can make a direct comparison to our previous work calculating the mass flux across the color-magnitude diagram. This approach will ultimately be used to tie together different epochs of the observed CMD by comparing the measured CMD flux to the measured changes in CMD with redshift.

We also calculate a past SFA as well (the SFA for the two time steps preceding the current one, SFJ, which stands for Star Formation Jerk (as in the derivative of the acceleration)). Note that the SFA and SFJ can be positive or negative. A negative SFA would signal a recent starburst, while a positive SFA would indicate on-going quenching of star formation. A positive SFA and SFJ would indicate a quench of longer duration.

The result is a matrix relating eight observables to the 10 physical parameters for each of 20 bins in $D_{n}(4000)$, and in nine redshift bins or $208 \times 11$ matrices. The matrix elements are denoted $M_{p, o, d, z}$ where $p$ refers to the physical parameter, $o$ to the observable, $d$ to the $D_{n}(4000)$ value, and $z$ to the coarse redshift bin. In Figure 6 we show some sample fits combined for all $D_{n}(4000)$ and redshift bins. We note that there is moderate error in the SFA fit as well as some bias. Fitting error is included in assessing the error in our mean SFA calculations. Biases are small and discussed in Appendix B.

Physical parameters are derived from

$$
P_{p}(\mathrm{est})=\sum_{o=1}^{o=8} M_{o, p, d, z} O_{o}
$$

or for the set of observables used here,

$$
\begin{aligned}
P_{p}(\mathrm{est})= & M_{1, p, d, z}(\mathrm{FUV}-\mathrm{NUV}) \\
& +M_{2, p, d, z}(\mathrm{NUV}-u)+M_{3, p, d, z}(u-g) \\
& +M_{4, p, d, z}(g-r)+M_{5, p, d, z}(r-i) \\
& +M_{6, p, d, z} D_{n}(4000)+M_{7, p, d, z} H_{\delta A} \\
& +M_{8, p, d, z} M_{i}+\text { constant. }
\end{aligned}
$$

A sample set of coefficients is given in Table 1 .

\subsection{Influence Functions}

In general not all observables used in the above fits are available. Some, such as $H \delta_{A}$, may be difficult to obtain. It is useful therefore to quantify the impact each observable has on each derived physical parameter. We do this by calculating the decrease in variance when using the observable relative to that when not using the observable. This is normalized to the total variance in the physical parameter over the full sample in a 
Table 1

Sample of Regression Coefficients with $D_{n}(4000)=1.30$ for $0.0<z<0.3$

\begin{tabular}{|c|c|c|c|c|c|c|c|c|c|}
\hline Parameter & $\beta$ & $\mathrm{NUV}-u$ & $u-g$ & $g-r$ & $\mathrm{NUV}-i$ & $D_{n}(4000)$ & $\mathrm{H} \delta_{a}$ & $M_{i}$ & Const \\
\hline$A_{\mathrm{FUV}}$ & -0.055 & -0.606 & 0.496 & 0.530 & 0.806 & -0.456 & 0.029 & -0.034 & -0.482 \\
\hline$(\mathrm{NUV}-r)_{0}$ & -0.113 & -0.281 & 0.274 & 0.334 & 0.559 & -0.340 & 0.018 & -0.037 & 0.037 \\
\hline SFA & 0.430 & 1.977 & 0.168 & -2.063 & -0.758 & 1.673 & 1.102 & -0.015 & -5.552 \\
\hline $\log (\mathrm{SFR})$ & -0.259 & -0.846 & 0.388 & 0.568 & 0.502 & -0.454 & -0.025 & -0.442 & 18.630 \\
\hline $\log$ (sSFR) & -0.244 & -0.616 & 0.255 & 0.332 & 0.279 & -0.436 & 0.016 & -0.027 & 0.903 \\
\hline $\log \left(M_{*}\right)$ & -0.015 & -0.230 & 0.133 & 0.236 & 0.223 & -0.018 & -0.041 & -0.415 & 17.727 \\
\hline$Z_{\text {gas }}$ & -0.039 & -0.143 & 0.114 & 0.115 & 0.137 & -0.040 & -0.035 & -0.336 & 12.459 \\
\hline$Z_{*}$ & -0.053 & -0.428 & 0.250 & 0.369 & 0.331 & 0.021 & -0.041 & -0.504 & 19.218 \\
\hline Ext. & 0.869 & 3.710 & 0.407 & -3.735 & -1.508 & 2.669 & 1.882 & -0.007 & -9.502 \\
\hline
\end{tabular}

Table 2

Influence Function

\begin{tabular}{|c|c|c|c|c|c|c|c|c|}
\hline Parameter & $\bar{\beta}$ & $\mathrm{NUV}-u$ & $u-g$ & $g-r$ & $\overline{\mathrm{NUV}}-i$ & $\overline{D_{n}(4000)}$ & $\overline{\mathrm{H} \delta_{a}}$ & $\overline{M_{i}}$ \\
\hline $\log \left(M_{*}\right)$ & 0.13 & 0.14 & 0.12 & 0.10 & 0.13 & 0.00 & 0.08 & $\overline{0.88}$ \\
\hline$A_{\mathrm{FUV}}$ & 0.12 & 0.29 & 0.32 & 0.31 & 0.38 & 0.00 & 0.01 & 0.02 \\
\hline$\Delta(\mathrm{NUV}-i)$ & 0.13 & 0.31 & 0.31 & 0.31 & 0.38 & 0.00 & 0.01 & 0.04 \\
\hline $\log (\mathrm{sSFR})$ & 0.49 & 0.28 & 0.16 & 0.17 & 0.20 & 0.01 & 0.14 & 0.22 \\
\hline SFA & 0.17 & 0.16 & 0.03 & 0.14 & 0.10 & 0.00 & 0.36 & 0.05 \\
\hline SFJ & 0.00 & 0.00 & 0.00 & 0.00 & 0.00 & 0.01 & 0.16 & 0.07 \\
\hline$Z_{\text {gas }}$ & 0.10 & 0.08 & 0.05 & 0.03 & 0.07 & 0.00 & 0.09 & 0.73 \\
\hline$Z_{*}$ & 0.13 & 0.14 & 0.13 & 0.11 & 0.13 & 0.00 & 0.10 & 0.86 \\
\hline
\end{tabular}

given $D_{n}(4000)$ bin:

$$
I[p, o, d, z] \equiv \frac{\left\langle\sigma_{[p, \bar{o}, d, z]}^{2}\right\rangle-\left\langle\sigma_{[p, o, d, z]}{ }^{2}\right\rangle}{\sigma_{[p, d, z]}^{2}}
$$

for physical parameter $p, D_{n}(4000)$ bin $d$, and observable $o$ either used $(o)$ or not used $(\bar{o})$. The mean is taken over all possible nontrivial combinations of observables (with or without observable $o$ ). A value of 1.0 would mean that the observable completely eliminates the parameter variance when introduced, and a value 0.0 means that the observable has no influence on the fit.

For example, for $D_{n}(4000)=1.40$, the influence function for $A_{\mathrm{FUV}}$ is $(0.24,0.34,0.30,0.30,0.49,0.07,0.10)$ for (FUV NUV, NUV $\left.-u, u-g, g-r, r-i, H \delta_{A}, M_{i}\right)$. Each photometric color makes a contribution to the reduction in fit variance, with NUV $-i$ reducing over $50 \%$ of the variance. Specific SFR ( $\log (\mathrm{sSFR})$, or $\log (\mathrm{SFR}) / M_{*}$, has influence functions $(0.54$, $0.29,0.06,0.08,0.20,0.17,0.20)$. The bulk of the information comes from FUV - NUV and NUV - $u$, with virtually no impact from $u-g$ or $g-r$. Finally, SFA has influence functions $(0.19,0.16,0.03,0.11,0.11,0.49,0.08)$. Most of the information comes from $H \delta_{A}$.

Table 2 gives the mean influence functions (averaged over all $\left.D_{n}(4000)\right)$. Figure 7 shows a color-coded display of the same information.

\subsection{Degeneracies/Observational Basis}

This method allows us to quantify parameter degeneracies in a simple fashion. Consider the seven-dimensional space of observations, and a single physical parameter $P_{i}$. A vector exists in this space in the direction that produces the maximum change in derived physical parameter. This is just the gradient in $P_{i}$, which is given by the matrix coefficients:

$$
\nabla \boldsymbol{P}_{i}=\sum_{o} M_{i, o} \hat{j}_{o}
$$

where $\hat{j}_{o}$ is a unit vector in the direction of the observable $o$ in this multidimensional space. The degeneracy of two physical parameters $P_{i}$ and $P_{j}$ can be determined from the dot product of these two gradients:

$$
D_{i, j} \equiv \frac{\nabla \boldsymbol{P}_{i} \bullet \nabla \boldsymbol{P}_{j}}{\left|\nabla \boldsymbol{P}_{i}\right|\left|\nabla \boldsymbol{P}_{j}\right|}
$$

A degeneracy of $D_{i, j}=1$ would mean that the two derived physical parameters come from the same linear combination of observables and are completely degenerate. Degeneracy can be negative, if two observables give the same information but with opposite dependences. Table 3 gives the degeneracy function. The degeneracies averaged over $D_{n}(4000)$ and redshift bins tabulated in Table 2 and displayed in Figure 8.

We note for example that the mass-weighted ages have a degeneracy of -0.72 , since both depend strongly on $H \delta_{A}$ (and 

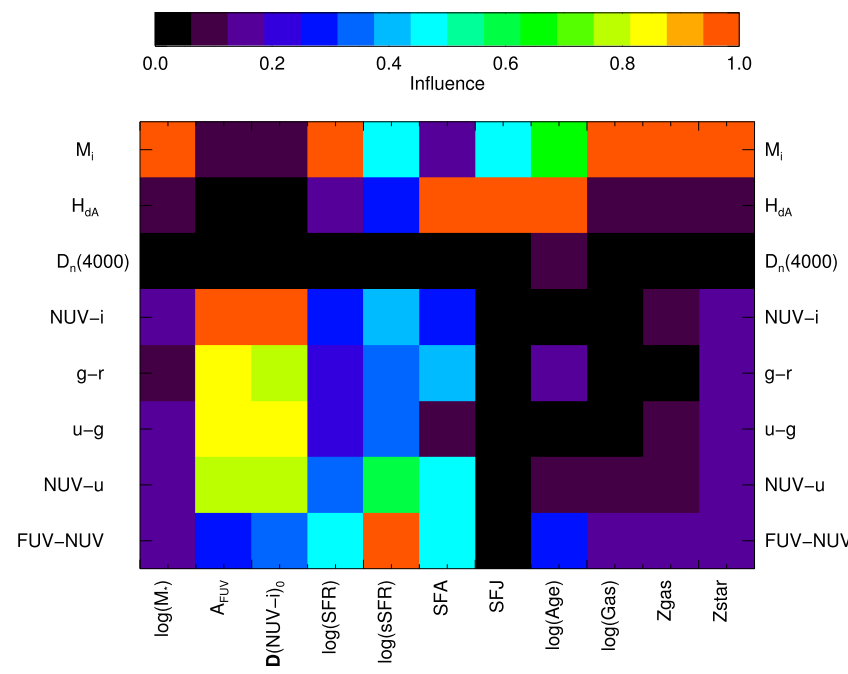

Figure 7. Influence functions for each parameter. This gives a graphic representative of the sensitivity of a given observable on recovering a given physical parameter (see text). The influence functions are normalized for each observable so that the observable with the maximum influence has 1.0. For example, $M_{i}$ has a strong influence on $\log \left(M_{*}\right), \log (\mathrm{SFR}), \log \left(M_{\mathrm{gas}}\right), Z_{\mathrm{gas}}$, and $Z_{*}$. Extinction and extinction-corrected NUV $-i\left[\Delta(\mathrm{NUV}-i)=(\mathrm{NUV}-i)_{0}-\right.$ $(\mathrm{NUV}-i)]$ are strongly influenced by NUV $-i, g-r, u-g$, NUV $-u$, and FUV - NUV.

$\left.D_{n}(4000)\right)$. This means that their influence vectors are $\sim 45^{\circ}$ apart. So while they are related they are not identical. It is not surprising there is degeneracy here. It may be counterintuitive that the degeneracy is negative, since one associates bursting with younger populations. However, this makes sense. The coefficients are calculated in a fixed $D_{n}(4000)$ bin. A galaxy with a smooth SFR will have a particular $H \delta_{A}$ associated with that $D_{n}(4000)$. If there was more SFR in the past (quenching), $H \delta_{A}$ will be higher than this smooth baseline since it peaks at hundreds of millions of years. The mass-weighted age will also be younger. If there was less SFR in the past (e.g., more in the present, bursting) then $H \delta_{A}$ will be lower than the baseline and the mass-weighted age will be greater.

In some sense all colors and spectral indices are "lightweighted ages" with different averaging kernels. For example, extinction-corrected NUV $-i$ is highly correlated with sSFR, since NUV tracks SFR (short-term light-weighted age) and the $i$-band has a very long averaging kernel and therefore is a tracer of stellar mass. Thus SFA is derived from color/index differences (see plots in Appendix A) that can be linearized within individual $D_{n}(4000)$ bins.

\subsection{Error Propagation/Observable Figure of Merit}

Since the derived parameters are linear functions of the observables, it is a simple matter to propagate observational errors to determine the total observational error component of the derived parameters. This can then be combined with the fitting error derived from the MLR step. If the observational error is large, and its influence is small, including the observation will actually increase the uncertainty of the derived parameter. Clearly the criterion for including an observable $o$ with an observational error $\sigma_{o}$ is

$$
\sigma_{[p, o, d, z]}^{2}+M_{o, p, d, z}{ }^{2} \sigma_{o}^{2}<\sigma_{[p, \bar{o}, d, z]^{2}} .
$$
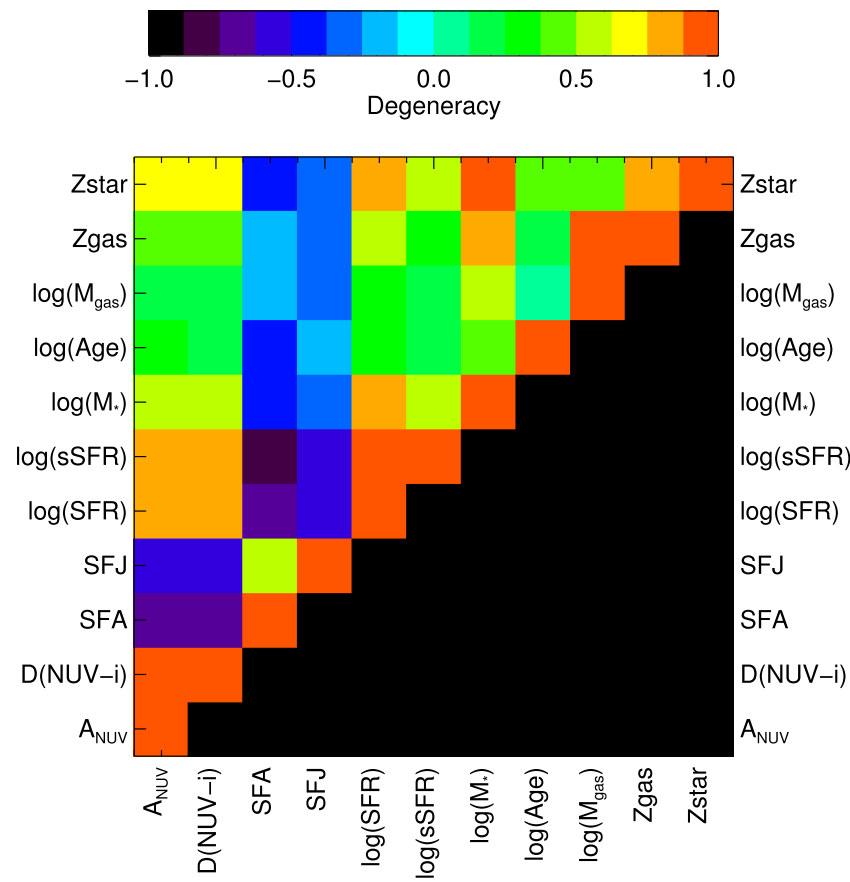

Figure 8. Degeneracy between derived physical parameters. A negative degeneracy implies that the parameters are inversely correlated. This gives a graphic representative of the degeneracy $D_{i, j}$ between parameters $i$ and $j$ (see text). A degeneracy of $D_{i, j}=1$ or $D_{i, j}=-1$ implies that the parameters cannot be independently extracted. For example, $\log \left(M_{*}\right)$ and $Z_{*}$ are highly degenerate, as are the change extinction correction to NUV $i\left(\Delta(\mathrm{NUV}-i)=(\mathrm{NUV}-i)_{0}-(\mathrm{NUV}-i)\right)$ and $A_{\mathrm{FUV}}$.

\section{Applications: GALEX/SDSS Galaxies}

Once we determine the matrix of linear coefficients, we can proceed to apply the method to real galaxies. We present this simply as an illustration of the potential of the methodology presented in this work, and expect that the full scientific yield will be realized over a range of studies and applications in the future.

\subsection{Observed Sample}

We use the same GALEX/SDSS spectroscopic sample as in Martin et al. (2007). Our sample is NUV-selected in the GALEX Medium Imaging Survey (MIS; Martin et al. 2005). The MIS/SDSS DR4 co-sample occupies $524 \mathrm{deg}^{2}$ of the north Galactic polar cap and the southern equatorial strip. Our sample is cut as follows: (1) NUV detection, nuv_weight $>800$; (2) SDSS main galaxy sample, $z_{\text {conf }}>0.67$ and specclass $=2$; (3) $14.5<r_{0}<17.6,16<\mathrm{NUV}<23.0 ;$ (4) nuv_artifact $<2 ;(5)$ field radius less than 0.55 ; (6) $0.02<z<0.22$. We use $D_{n}(4000)$ and $H \delta_{A}$ as calculated and employed for the SDSS spectroscopic sample by Kauffman et al. (2003) and available as the MPIA/JHU DR4 Value-Added Catalog. $H \delta_{A}$ is corrected for nebular emission. The sample properties, galactic extinction and $k$-correction, and cuts are discussed further in M07.

There are slight differences in the mean colors of the observed sample with respect to the model colors. These are typically $\sim 0.1 \mathrm{mag}$ but rise to $\sim 0.6 \mathrm{mag}$ in the case of NUV $-u$ for several bins in $D_{n}(4000)$. Also, model $H \delta_{A}$ are higher than observed $H \delta_{A}$ by about 0.5 over a range of $D_{n}(4000)$. Model color dispersions are comparable to the observed dispersions 
(a)

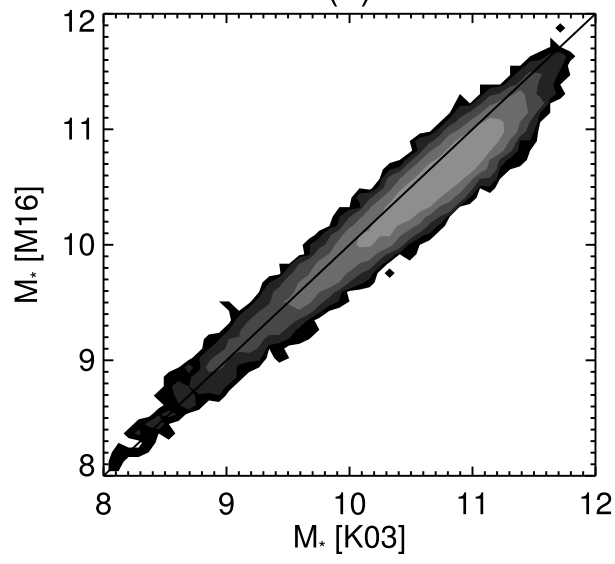

(c)

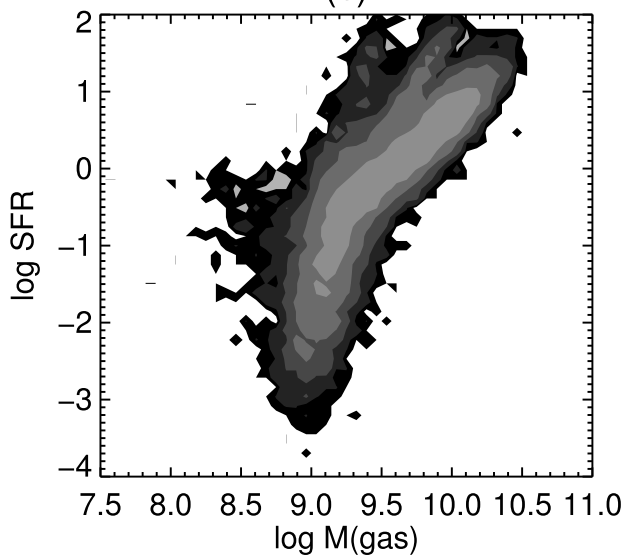

(b)

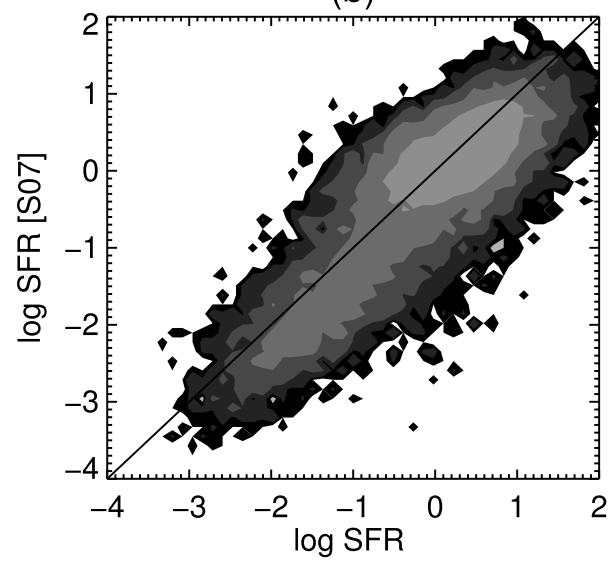

(d)

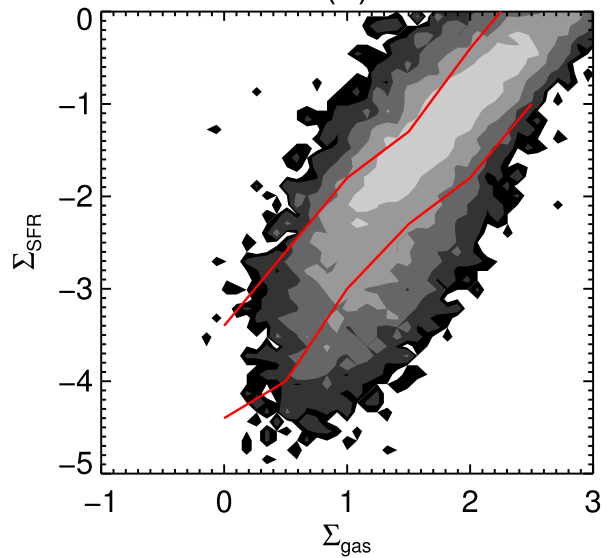

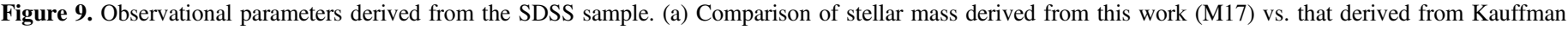

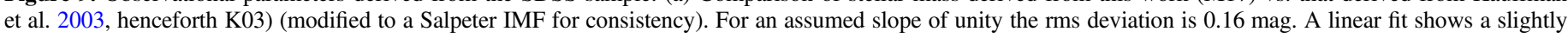

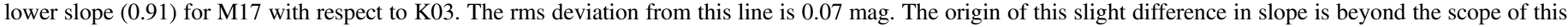

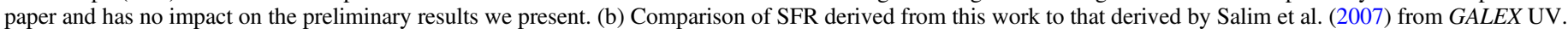

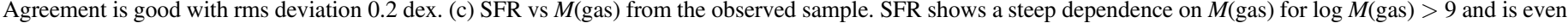

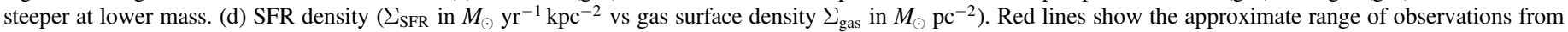
Bigiel et al. (2008) and Wyder et al. (2009).

when observation errors are included. The model mean colors in each $D_{n}(4000)$ bin have been adjusted to match the observed mean colors prior to fitting a model in order to ensure that the range of derived parameters is not outside the bounds of the fitted parameters. See Appendix A for further details.

We compare the stellar mass derived by our new approach to that derived by Kauffman et al. (2003) in Figure 9(a). The derived masses compare well, with an rms deviation of $\sim 0.16$ dex around a slope of unity. We also find some evidence for slightly different masses at low and high values as indicated by a best-fit slope of 0.9 for the comparison. This small difference does not affect our preliminary findings discussed below. We compare in Figure 9(b) our SFR to the SFR derived by Salim et al. (2007) also from GALEX UV and an otherwise independent method. The agreement is good with rms deviation of 0.22 dex (comparable to the scatter found by Salim et al. (2007) of 0.17). We show the SFR versus $M$ (gas) that we derive in Figure 9(c). Finally, we show the globally averaged SFR density versus gas mass density (the standard Schmidt-Kennicutt law, see Kennicutt 1998), compared to results from Bigiel et al. (2008) for local galaxies.

\subsection{Application 1: Quenching and Starbursts in the Green Valley}

One of our main goals with this technique is to understand the transition of galaxies between the star-forming blue sequence (or "main sequence") and the passively evolving red sequence. In previous papers (Martin et al. 2007; Gonçalves et al. 2012) we have evaluated the timescales required for a galaxy to quench star formation and complete the transition from blue to red, both at low redshifts $(z \sim 0.1$; Martin et al. 2007) and at intermediate ones $(z \sim 0.8$; Gonçalves et al. 2012), using a combination of the NUV $-r$ color and the spectroscopic indices $D_{n}(4000)$ and $H \delta_{A}$. Nevertheless, those papers assume a simplistic model of star formation histories in which galaxies move single-handedly from blue to red sequence with exponentially declining star formation rates. We do know, however, that some galaxies of intermediate color are actually bursting, getting temporarily bluer perhaps due to a sudden inflow of gas and subsequent star formation episode (e.g., Rampazzo et al. 2007; Thilker et al. 2010; Thomas et al. 2010; Fang et al. 2012; Salim et al. 2012).

Recognizing this two-way flow, the SFA is an appropriate measure of the rate of color evolution across the green valley. 


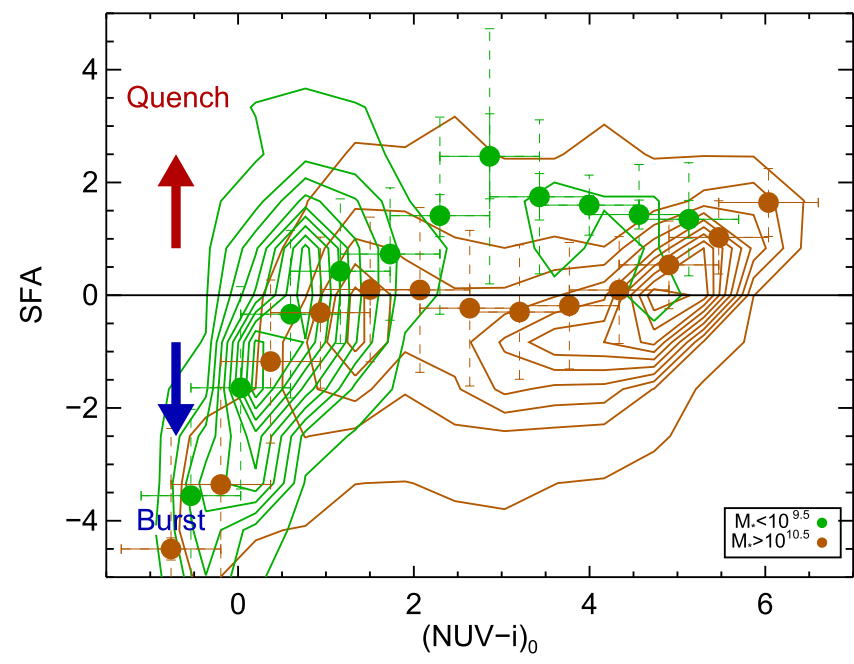

Figure 10. Star formation acceleration (SFA) vs. (NUV $-i)_{0}$ for SDSS galaxies in two mass bins cut at transition mass $M<10^{9.5} M_{\odot}$ and $M>10^{10.5} M_{\odot}$. Contours show the distribution of galaxies in the two mass bins. Dots and error bars show mean SFA and error in color bins. The following trends are apparent. Bluer galaxies of both blue and red sequences have bursting SFAs, while redder galaxies of both sequences tend toward quenching SFA. Also, at all (NUV $-i)_{0}$ colors, on average, lower-mass galaxies have higher SFA (more quenching) than higher-mass galaxies.

Again, SFA is positive for quenching galaxies and negative for galaxies undergoing starbursts. Figure 6 shows the result for SFA for model galaxies and Figure 8 shows the observable influence function.

We applied this to the identical set of galaxies used in Martin et al. (2007), and Figure 10 shows the resulting SFA versus extinction-corrected NUV $-i$ color in two mass bins. Several phenomena can be seen in this figure. Ignoring mass dependence for the moment, blue-sequence galaxies show colors correlated with their SFA-the bluest galaxies have negative, "bursting" SFAs, while redder blue-sequence galaxies are "quenching." The red sequence has a similar "tilt" in the diagram: the bluest galaxies have negative, "bursting" SFAs, while redder red-sequence galaxies are "quenching." The origin of some of the spread in both sequences can be ascribed to recent changes in the SFR.

We can plot the color derivatives on the diagram of sSFR versus stellar mass. We show this in Figure 12. This diagram represents a first attempt to capture the "flow" of galaxies on the color-magnitude diagram (or equivalent sSFR-mass diagram). In this diagram red arrows represent average quenching and blue average bursting for galaxies in each sSFR-mass bin. The total length of the two arrows is proportional to the rms spread of the SFA, while the relative proportion of red and blue depends on the mean SFA (see caption). The head of each arrow corresponds to the current mass-sSFR, while the tail is the previous location on the diagram scaled to roughly $100 \mathrm{Myr}$ in the past. We can also calculate the mean SFA in each sSFRmass bin. This is shown in Figure 11.

In M07 we reported a measurement of the mass flux of galaxies across the green valley as an upper limit, because we used a simple monotonic quenching model to derive the color derivative ( $d y / d t$, now relabeled SFA). Using the same sample but revising the color derivative in each mass bin, we can calculate the true mass flux from blue to red taking into account net bursting and quenching. The revised flux versus mass is

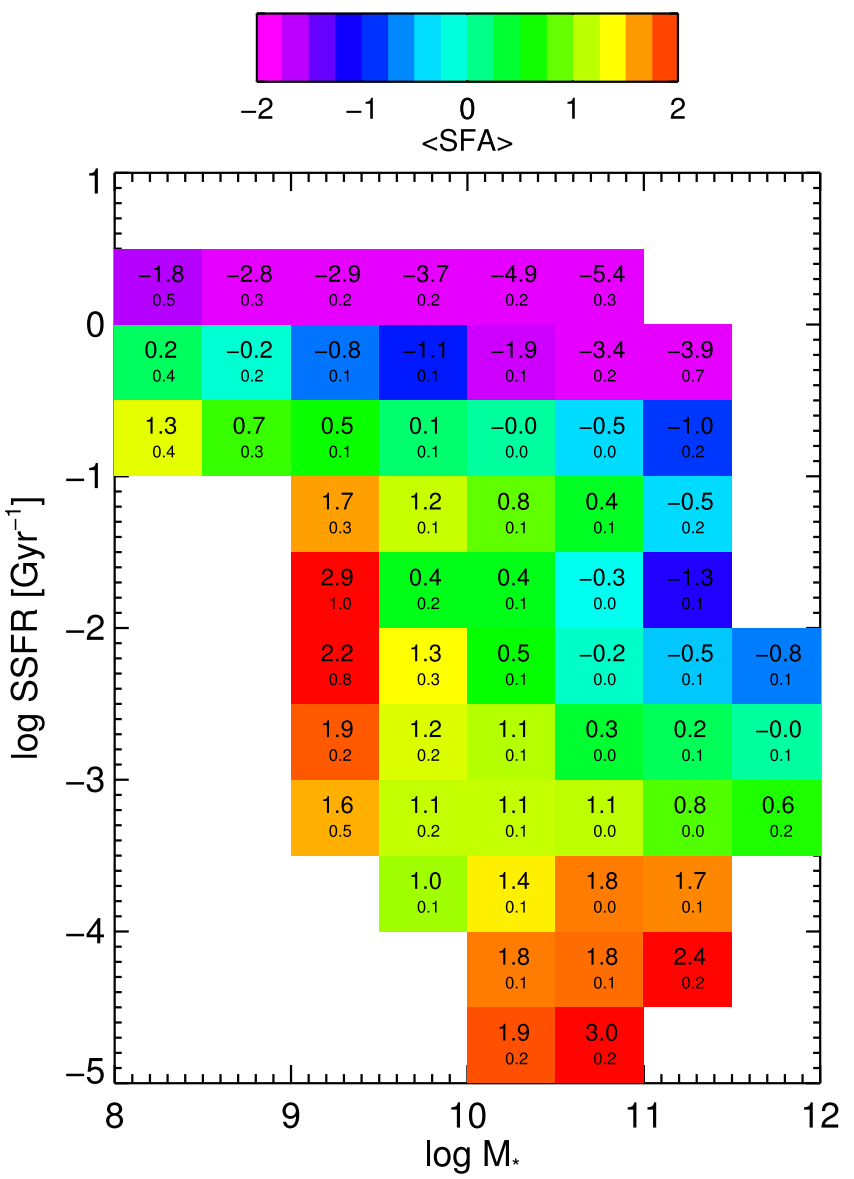

Figure 11. Mean star formation acceleration (SFA) indicated by color on the diagram of $\log (\mathrm{sSFR}) \mathrm{vs}$. $\log M_{*}$ for SDSS galaxies. The large number in a box is the mean SFA, the small number is the standard error of the mean.

given in Table 4. Our new mass flux (calling this method 4 to maintain continuity with the three methods presented in M07) is $\dot{\rho}_{\mathrm{BR}}=(2.3 \pm 0.07) \times 10^{-2} M_{\odot} \mathrm{yr}^{-1} \mathrm{Mpc}^{-3}$. It is entirely consistent with the value derived by $\mathrm{M} 07$, and also with the estimates based on the mass evolution of the blue sequence (Blanton 2006; Martin et al. 2007) and red sequence (Faber et al. 2007). We plot this result in Figure 13.

Now consider the dependence on stellar mass. Lower-mass galaxies in the green valley are mostly quenching, while higher-mass galaxies are both quenching and bursting. This is demonstrated in the sSFR-mass diagrams of Figures 12 and 14. In Figure 14 we have calculated average SFA and display it versus specific SFR (sSFR) for two mass cuts. The mean SFA is 1-3 higher for galaxies with $M_{*}<10^{9.5} M_{\odot}$ than for galaxies with $M_{*}>10^{10.5} M_{\odot}$. A plausible scenario for this is given in Figure 15: lower-mass galaxies are accreting and becoming satellite galaxies, having their star-forming gas tidally and/or ram-pressure stripped, while higher-mass galaxies are receiving this gas and reacting with new star formation. These mass differences are extremely important for galaxy models, and obtaining significant numbers of low-mass green-valley galaxies and comparing them to high-mass galaxies requires an analysis of a larger SDSS/GALEX data set.

It is interesting to compare these observed results to the predictions of the semianalytic models used to generate the star formation histories and parameter coefficients. As we discuss in Appendix B, the models predict trends that are qualitatively 
(a)

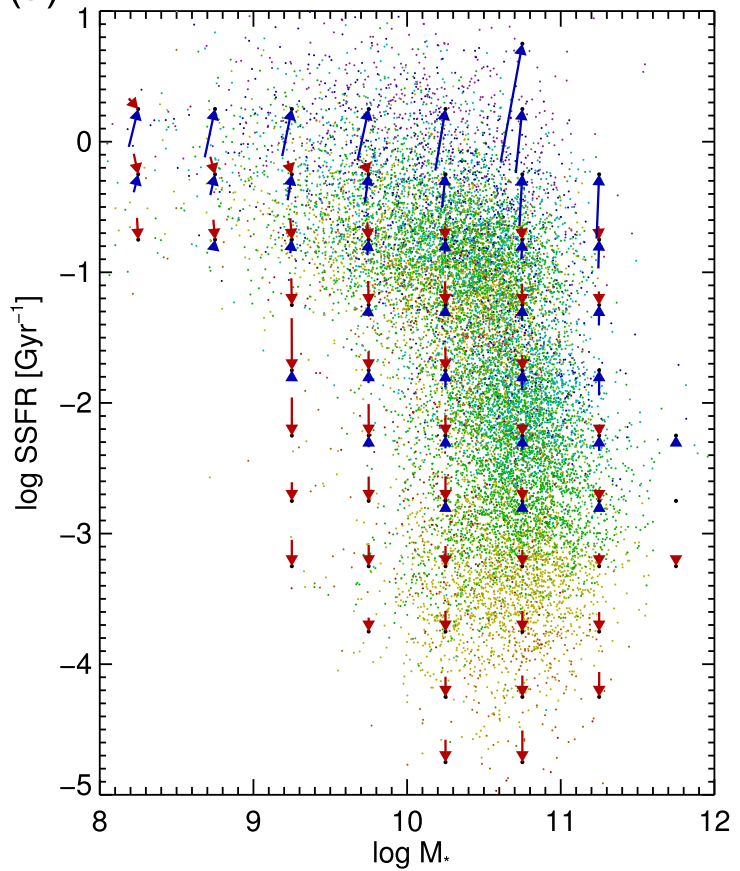

(b)

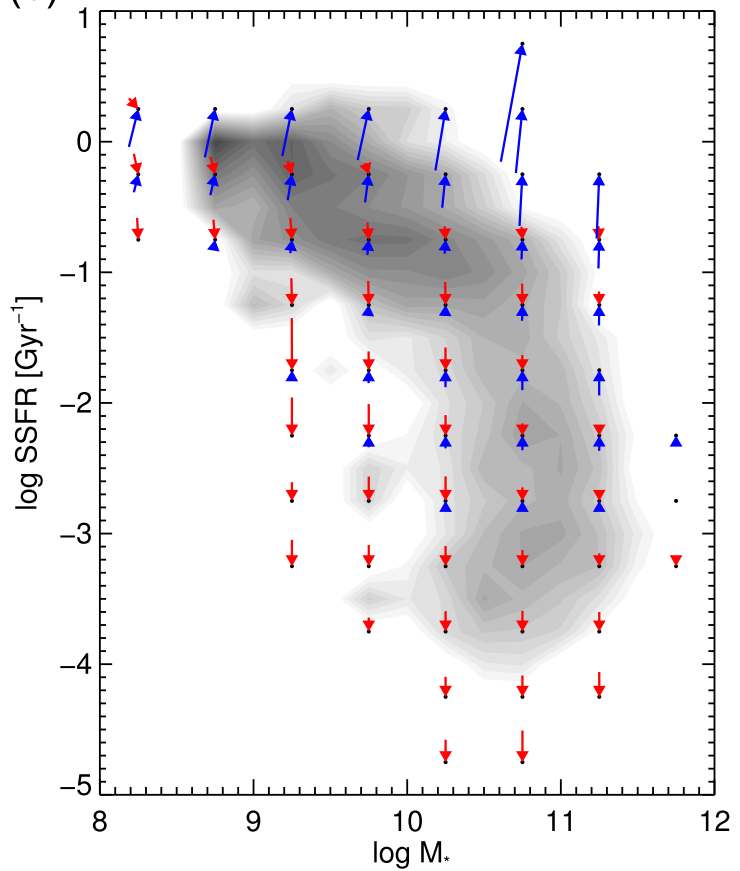

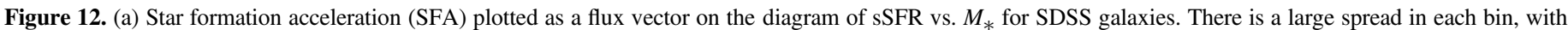

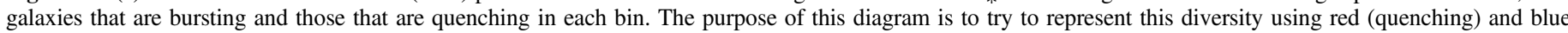

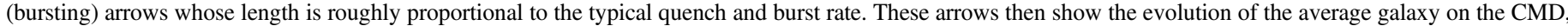

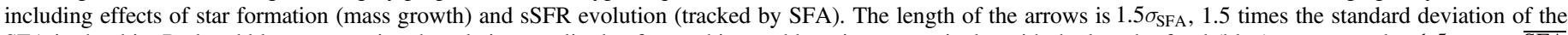

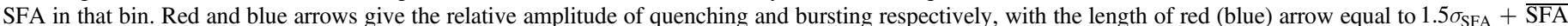

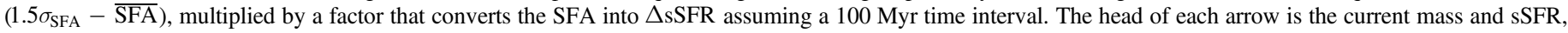

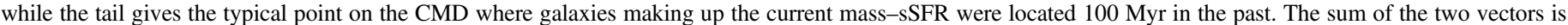

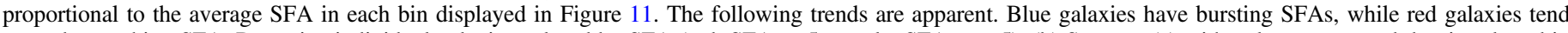

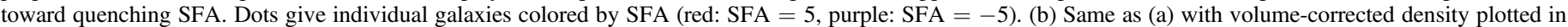

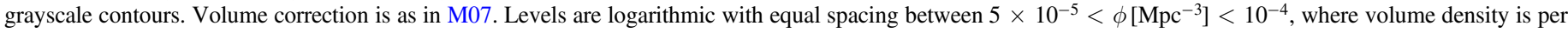
unit 0.5 dex bin in $\log M_{*}$ and $\log (\mathrm{sSFR})$.

similar but quantitatively much weaker than those we observe. The observed results are quite distinct from the model predictions.

\subsection{Application 2: The AGN/SFA Connection}

AGNs are potentially powerful sources of feedback that could accelerate quenching and maintain galaxies on the red sequence (Croton et al. 2006; Martin et al. 2007; Nandra 2007; Schawinski et al. 2009). Furthermore, there is growing evidence that quenching (especially at high stellar masses) might be related to the growth of stellar density in the central part of the galaxy, probably due to AGN activity and concomitant bulge growth (e.g., Cheung et al. 2012; Mancini et al. 2015). As Figure 16 shows, AGNs preferentially occupy the green valley. We would like to attempt to answer a simple physical question: All else being equal, does the presence of an AGN accelerate quenching in transition galaxies? There is preliminary evidence for this, which we show in Figure 17. At intermediate sSFR, the presence of an AGN appears to accelerate quenching by roughly a factor of 2-3. This would appear to support a scenario in which the presence of an AGN might also be connected with a starburst event (e.g., King 2005; Gaibler et al. 2012; Rovilos et al. 2012), and only unequivocally quenches star formation at later stages, when feedback drives the gas away (Di Matteo et al. 2005; Springel et al. 2005).

However, a large, statistically robust sample is required to confirm this tentative conclusion. AGN fraction correlates with many other properties, and it must be established that these correlations do not artificially create this dependence. The larger GALEX Legacy Survey/SDSS sample will allow us to test this dependence while other correlates are held fixed, and even investigate whether there is a relation between quenching timescales and AGN luminosities. One of the future goals of this study is to firmly establish whether AGNs accelerate quenching, and under what circumstances.

Our preliminary results can also be used to place some constraints on the formation of the most massive $\left(M_{*}>\right.$ $\left.10^{11.5} M_{\odot}\right)$ quiescent galaxies. Let's use a cut of $\log (\mathrm{sSFR}$ $\left.\left(\mathrm{Gyr}^{-1}\right)\right)=-2$ to separate star-forming and quiescent systems (used in the literature and evident from the distribution of galaxies in Figure 12). If the most massive $\left(M_{*}>10^{11.5} M_{\odot}\right)$ quiescent galaxies are the result of dry mergers between already quiescent less massive systems, then in principle there should not be a change in their SFA. However, we clearly see in Figure 11 that even the most massive quiescent systems show some degree of bursting. Wet mergers can qualitatively explain the bursting phase for them. The most bursting is happening in the most massive star-forming systems as seen in Figures 11 and 14. These starforming systems are likely going through wet major mergers that result in gas in their outskirts falling toward the center and getting compressed, causing the burst of star formation. Very massive star-forming systems $\left(M_{*}>10^{11.5} M_{\odot}\right.$ and $\left.\log (\mathrm{sSFR})>-0.5\right)$ are rare (see, e.g., Figure 11) because they have already been quenched and moved to the massive quiescent population likely through wet major mergers of less massive star-forming systems. 
Table 3

Degeneracy Function

\begin{tabular}{|c|c|c|c|c|c|c|c|c|c|c|c|}
\hline Parameter & $A_{\mathrm{FUV}}$ & $\Delta(\mathrm{NUV}-i)_{0}$ & SFA & SFJ & $\log (\mathrm{SFR})$ & $\log$ (sSFR) & $\log \left(M_{*}\right)$ & $\log ($ age $)$ & $\log \left(M_{\mathrm{gas}}\right)$ & $Z_{\mathrm{gas}}$ & $Z_{*}$ \\
\hline$\overline{A_{\mathrm{FUV}}}$ & 1.00 & 0.98 & -0.68 & -0.54 & 0.87 & 0.86 & 0.61 & 0.25 & 0.17 & 0.40 & 0.66 \\
\hline$\Delta(\mathrm{NUV}-i)$ & -1.00 & 1.00 & -0.64 & -0.53 & 0.84 & 0.82 & 0.59 & 0.20 & 0.19 & 0.41 & 0.63 \\
\hline SFA & -1.00 & -1.00 & 1.00 & 0.62 & -0.74 & -0.76 & -0.46 & -0.43 & -0.16 & -0.25 & -0.47 \\
\hline $\log (\mathrm{SFR})$ & -1.00 & -1.00 & -1.00 & -1.00 & 1.00 & 0.92 & 0.79 & 0.29 & 0.37 & 0.58 & 0.81 \\
\hline $\log (\mathrm{sSFR})$ & -1.00 & -1.00 & -1.00 & -1.00 & -1.00 & 1.00 & 0.50 & 0.16 & 0.20 & 0.35 & 0.55 \\
\hline $\log \left(M_{*}\right)$ & -1.00 & -1.00 & -1.00 & -1.00 & -1.00 & -1.00 & 1.00 & 0.42 & 0.56 & 0.79 & 0.98 \\
\hline$Z_{\mathrm{gas}}$ & -1.00 & -1.00 & -1.00 & -1.00 & -1.00 & -1.00 & -1.00 & -1.00 & -1.00 & 1.00 & 0.77 \\
\hline$Z_{*}$ & -1.00 & -1.00 & -1.00 & -1.00 & -1.00 & -1.00 & -1.00 & -1.00 & -1.00 & -1.00 & 1.00 \\
\hline
\end{tabular}

Table 4

Mass Flux Table Using Method 4

\begin{tabular}{|c|c|c|c|c|c|c|c|}
\hline$M_{r}$ & $\log M_{*}$ & $\phi$ & \# & $\overline{d y / d t}(\mathrm{M} 07)$ & $\overline{d y / d t}$ & $\dot{\rho}_{\mathrm{BR}}$ & $\sigma\left(\dot{\rho}_{\mathrm{BR}}\right)$ \\
\hline $\begin{array}{l}-23.75 \\
\end{array}$ & 11.63 & $1.84 \times 10^{-7}$ & 5 & 0.82 & 0.82 & $1.3 \times 10^{-4}$ & $1.8 \times 10^{-5}$ \\
\hline-23.25 & 11.49 & $1.08 \times 10^{-6}$ & 22 & 0.70 & 0.69 & $4.6 \times 10^{-4}$ & $6.2 \times 10^{-5}$ \\
\hline-22.75 & 11.32 & $6.53 \times 10^{-6}$ & 100 & 0.85 & 0.54 & $1.5 \times 10^{-3}$ & $1.9 \times 10^{-4}$ \\
\hline-22.25 & 11.12 & $2.20 \times 10^{-5}$ & 217 & 0.87 & 0.53 & $3.1 \times 10^{-3}$ & $3.2 \times 10^{-4}$ \\
\hline-21.75 & 10.93 & $4.02 \times 10^{-5}$ & 252 & 0.94 & 0.61 & $4.2 \times 10^{-3}$ & $3.2 \times 10^{-4}$ \\
\hline-21.25 & 10.73 & $5.63 \times 10^{-5}$ & 211 & 0.87 & 0.69 & $4.2 \times 10^{-3}$ & $2.5 \times 10^{-4}$ \\
\hline-20.75 & 10.50 & $7.42 \times 10^{-5}$ & 154 & 1.05 & 0.81 & $3.8 \times 10^{-3}$ & $2.8 \times 10^{-4}$ \\
\hline-20.25 & 10.25 & $6.80 \times 10^{-5}$ & 77 & 1.37 & 0.93 & $2.3 \times 10^{-3}$ & $2.0 \times 10^{-4}$ \\
\hline-19.75 & 10.00 & $6.94 \times 10^{-5}$ & 40 & 1.69 & 1.17 & $1.6 \times 10^{-3}$ & $2.5 \times 10^{-4}$ \\
\hline-19.25 & 9.85 & $6.06 \times 10^{-5}$ & 21 & 1.50 & 1.32 & $1.1 \times 10^{-3}$ & $2.0 \times 10^{-4}$ \\
\hline-18.75 & 9.52 & $7.22 \times 10^{-5}$ & 9 & 2.90 & 1.22 & $5.8 \times 10^{-4}$ & $1.4 \times 10^{-4}$ \\
\hline Sum & & & & & & $2.3 \times 10^{-2}$ & $7.4 \times 10^{-4}$ \\
\hline
\end{tabular}

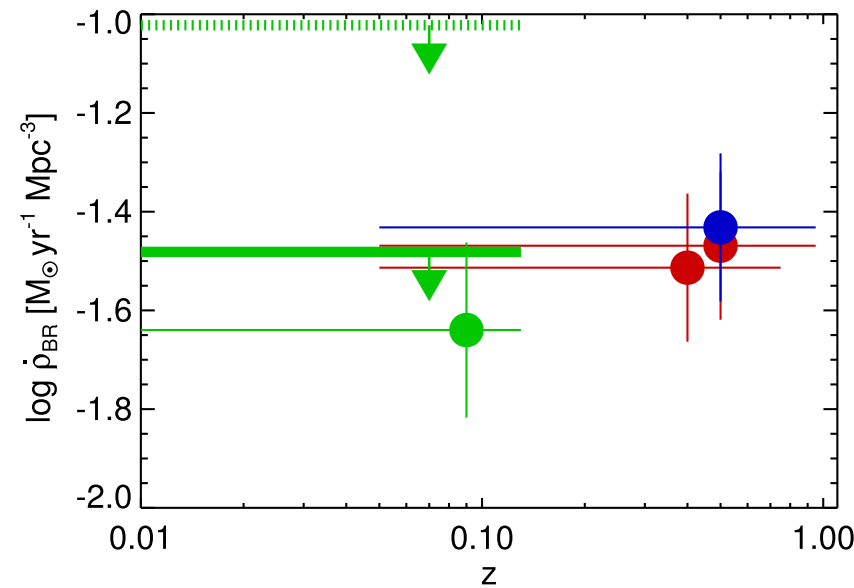

Figure 13. Total mass flux across the green valley (green dot with error bars) estimated using SFA and the galaxy sample of M07. The green dashed line shows the result of M07 method 1 (no extinction correction, color derivative calculated for the mean of all galaxies in the color-magnitude bin based on monotonically quenching star formation histories); the green solid line shows the result of M07 method 3 (extinction correction, color derivative calculated for each galaxy based on monotonically quenching star formation histories). Both were interpreted in M07 as upper limits because of the possible presence of bursting galaxies. Red points show mass flux estimated from the red sequence evolution of Faber et al. (2007). The higher point is based on evolution over $0<z<1$, and the lower on $0<z<0.8$. The blue point shows the estimate $-\dot{\rho}_{B}$ based on blue sequence evolution (derived in M07).

Therefore, part of the evolution of the most massive quiescent galaxies $\left(M_{*}>10^{11.5} M_{\odot}\right)$ is due to wet major mergers of less massive star-forming systems. We note that wet minor mergers

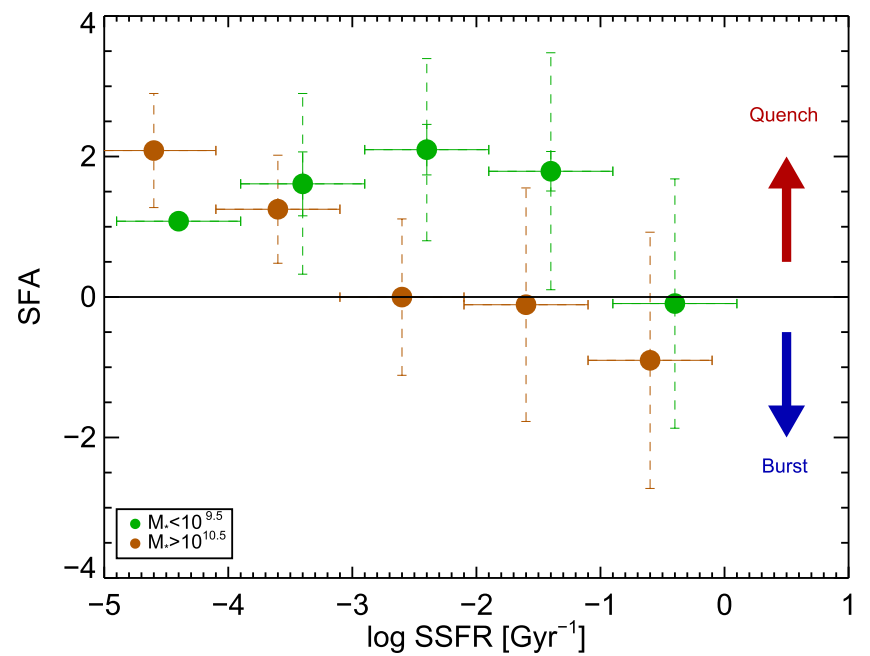

Figure 14. Average star formation acceleration (SFA) in several sSFR bins for SDSS galaxies in two mass bins cut at transition mass $M_{c}=10^{10} M_{\odot}$. As in Figure 10, the following trends are apparent. High sSFR galaxies have bursting SFAs, while low sSFR galaxies tend toward quenching SFA. For log (sSFR) $<-1.5 \mathrm{Gyr}^{-1}$ lower-mass galaxies have higher SFA (more quenching) than higher-mass galaxies, which average zero quenching (equal numbers of quenching and bursting galaxies). Dashed lines show the full range of the distribution, while solid vertical error bars show the resulting standard error of the mean SFA.

can have a similar effect too, without changing the mass of the massive quiescent galaxies much. Star formation jerk (SFJ) and a larger sample can potentially help distinguish between these scenarios. 


\section{Low Mass Satellites - Quenching by Tidal Stripping}
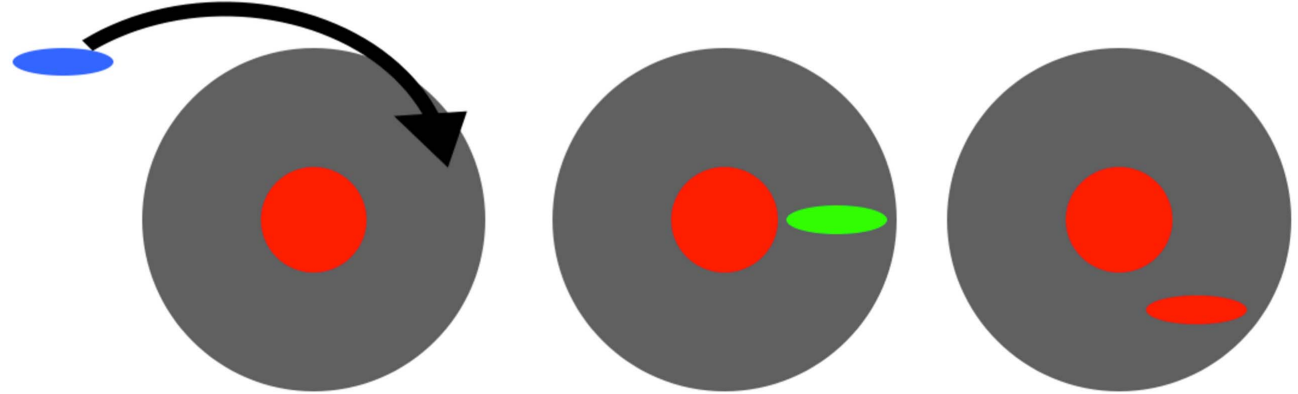

High Mass Centrals - Bursting from Accretion of New Gas-rich Satellites or IGM
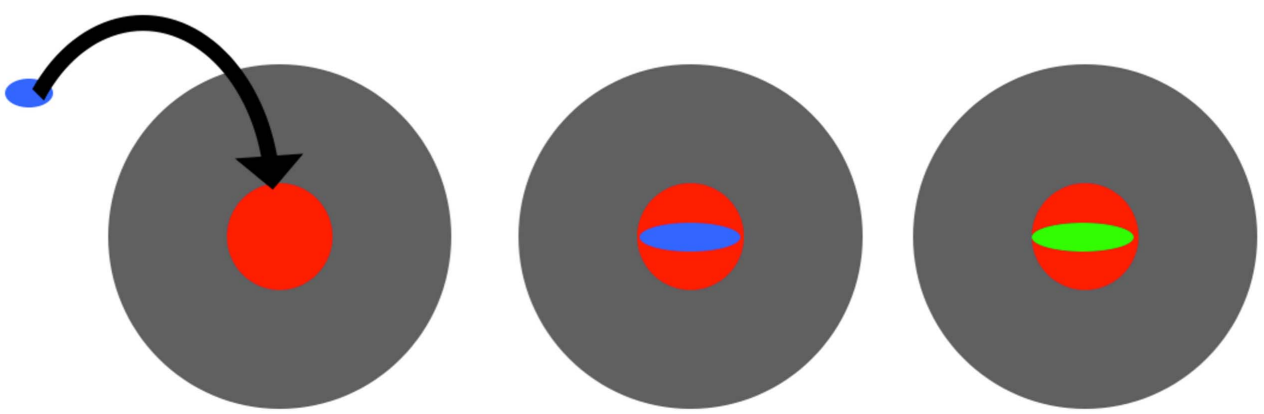

Figure 15. Cartoon model for results displayed in Figures 10-14. Low-mass galaxies are stripped as they enter higher-mass halos and are tidally or ram-pressure stripped of gas and quench. High-mass galaxies are preferentially central galaxies and occasionally suffer bursts from accretion events (merging satellites or infalling circumgalactic gas).
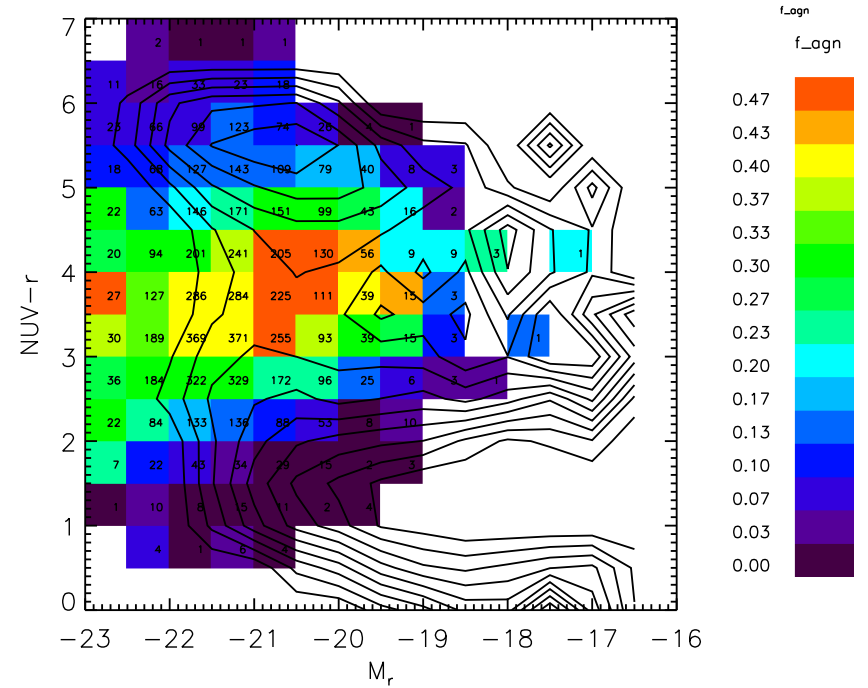

Figure 16. Color-magnitude diagram (contours in NUV $-r$ vs. $M_{r}$, extinction corrected) from Martin et al. (2007). AGN fraction in each color-magnitude bin shows that AGNs mostly occupy the green valley.

Interestingly, wet major mergers might also explain what we see in Figure 17 for AGNs. Wet major mergers tend to rejuvenate the nuclear activity but with some time delay after the star-bursting phase (due to star formation). According to Figure 17, for high sSFR values (star-forming phase), both AGN and non-AGN hosts are bursting (in the star formation phase of a merger), but after a while, they enter the quenching phase with AGN hosts showing higher quenching possibly due to the revived nucleus (as mergers cause the gas to funnel toward the nucleus), which is subsequently followed by outflows/ feedback to help quench galaxies more effectively. SFJ contains

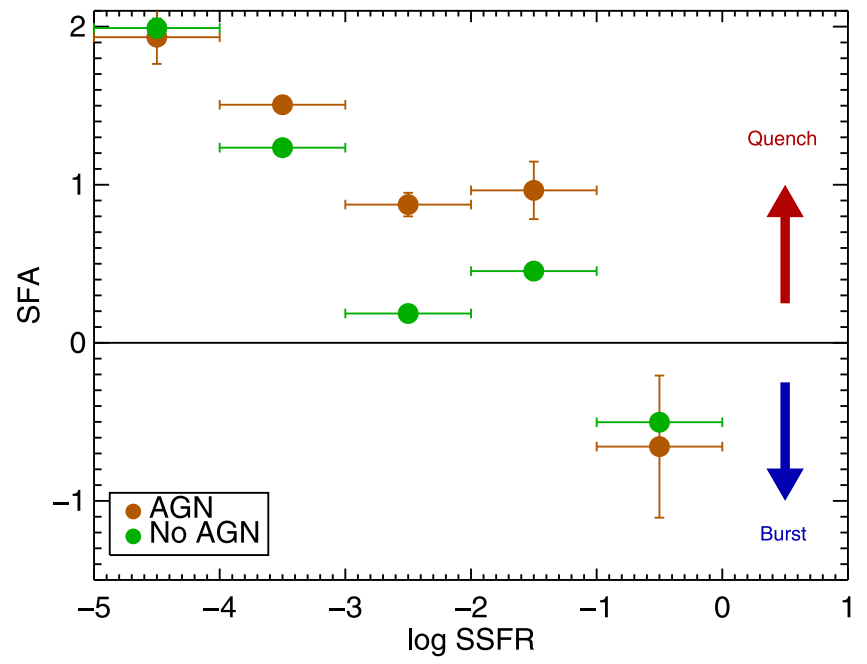

Figure 17. Average Star formation acceleration (SFA) in several sSFR bins for SDSS galaxies for AGNs and non-AGNs. As in Figure 14, SFA increases at lower sSFR. Since low-mass galaxies dominate the number density, the average is net quenching at low sSFR. Galaxies with an AGN show detectably higher SFA (quenching) than galaxies without. Vertical error bars show the standard error of the mean SFA.

information about the timescale of quenching/bursting events and can potentially be used to constrain this picture. We will study this in more details in a subsequent paper.

\section{Discussion and Summary}

\subsection{Issues and Caveats}

Aperture and Volume Effects. SDSS spectroscopy is obtained with 3 arcsecond fibers, which often do not subsume the full galaxy. M07 discussed this effect and dismissed it as 


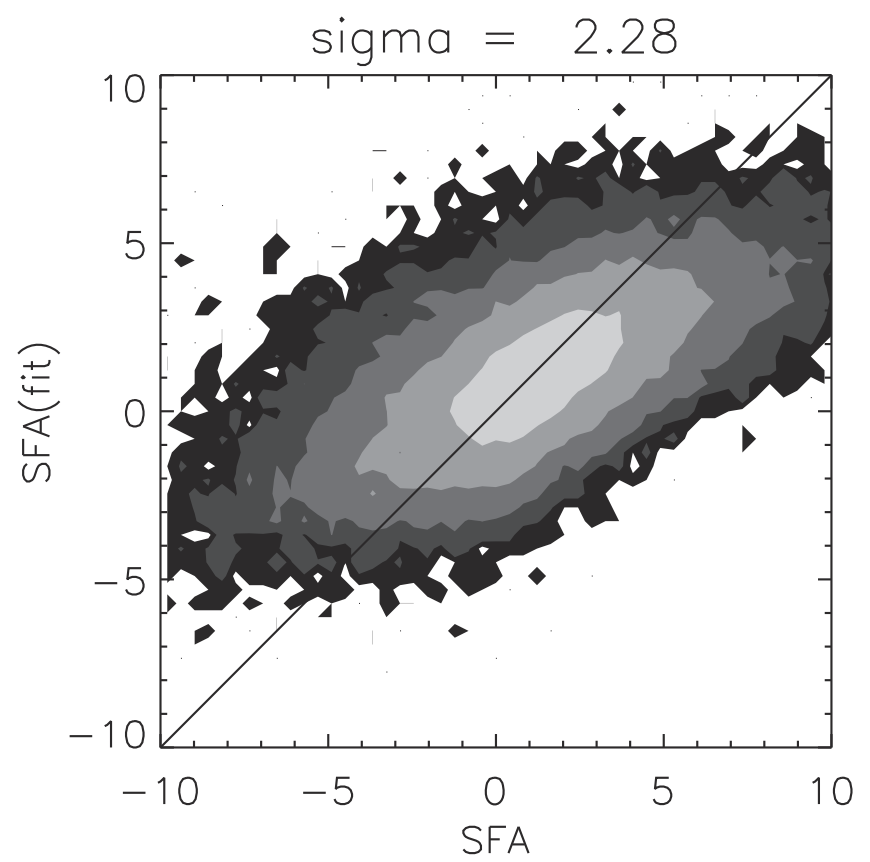

Figure 18. Recovered SFA vs model SFA in the test sample for which a large random SFR component has been added to decouple the star formation history from other physical parameters that affect observables such as extinction (compare to Figure 6(f)). The ratio of fitting error $(\sigma=2.28)$ relative to spread of SFA is approximately the same as in the reference models.

not significant, mainly on the strength of no detected dependence on average redshift. There are small variations in $\langle\mathrm{SFA}\rangle$ versus redshift that may be correlated with large-scale structure. There is no trend with increasing redshift. The mass trend of SFA does not diminish when the redshift range is restricted to $0.05<z<0.10$. This indicates that neither aperture, color selection, mass selection, nor volume effects explain the mass trend.

Extinction. We considered a number of variants of the extinction law behavior to determine whether our approach impacted the extraction of the star formation history. In all cases the derived extinction has sensible dependence on SFR, sSFR, metallicity, and gas mass. As we noted above, even when the extinction law is permitted to vary randomly between Milky Way and Calzetti, the rms error in $A_{\mathrm{FUV}}$ rises only to $\sim 0.5$ mag. Other than making subtle changes in the distribution of galaxies in the mass-SFR and mass-sSFR diagrams, the details of the extinction correction do not significantly impact SFA. We defer to a future paper a comparison of the extinction correction with direct methods that use the MIR/FIR and FUV/NUV luminosity.

Model Biases. It is important to ascertain whether the particular SAMs we have chosen to generate star formation histories are biasing the results for the observed SDSS sample. As we mentioned earlier, we believe that the SAMs provide a space of possible star formation histories, and if those histories span a similar space to actual galaxies (not necessarily with the same demographics), then the SFA we derive will not be sensitive to the models. We show in Appendix B that the SAMs give a quantitatively different SFA versus mass and sSFR than the observed galaxies.

We experimented with changing the star formation histories in the SAMs by adding a large random component (by replacing $\mathrm{SFR}$ with $2 \times \mathrm{SFR} \times r$ where $0<r<1$ is a uniform random deviate). The purpose of this was to show that recovery of the SFA is tied to star formation history alone and not some other observable quantity (such as extinction) given by the models. Even here we still recover the relationship between observables and SFA with similar coefficients and a similar ratio of fit noise to the total dispersion in SFA (which in this case is larger because of the very noisy star formation histories). This fit is shown in Figure 18. This occurs in spite of the large decoupling between the star formation histories and the other physical parameters in the models with a random star formation history. Just as SFR (the derivative of stellar mass) is traced by FUV, NUV, or $\mathrm{H} \alpha$ (extinction corrected) in a modelindependent way, so does SFA (effectively the derivative of $\log$ (sSFR)) trace the color derivative in an essentially modelindependent way. The caveat to this discussion is metallicity, which we turn to next.

Metallicity. Spectral indices and photometric colors are dependent on the metallicity of the stars producing them as well as on the star formation histories. As we discussed in Section 2.2.1, model metallicities are incorporated following the SAM metallicity evolution for each galaxy. Thus to first order metallicity effects are accounted for, to the extent that the metallicity evolution of the model galaxies matches that of the observed galaxies. We have checked to see whether uncorrected metallicity variations in the $H \delta_{A}-D_{n}(4000)$ relation can produce the mass trends that we observe. Consider an sSFR range of $-3<\log (\mathrm{SSFR})<-2$ in three mass bins $\left(9<\log M_{*}<10, \quad 10<\log M_{*}<11\right.$, and $11<\log M_{*}<$ 12). These give mean $D_{n}(4000)$ of $1.54,1.70$, and 1.76 . Using the mass-metallicity relation of Tremonti et al. (2004) and the $H \delta_{A}$-metallicity variation for fixed $D_{n}(4000)$ from Bruzual \& Charlot (2003), we can calculate $d H \delta_{A} / d\left(\log M_{*}\right)$, and using the fitting coefficients $d \mathrm{SFA} / d H \delta_{A}$ we find a spurious slope of $d$ SFA $/ d\left(\log M_{*}\right)=-0.5$ between $9<\log M_{*}<10$ and $10<$ $\log M_{*}<11, \quad$ and $\quad d$ SFA $/ d\left(\log M_{*}\right)=+0.15 \quad$ between $10<\log M_{*}<11$ and $11<\log M_{*}<12$. This should be compared with the observed $d \mathrm{SFA} / d\left(\log M_{*}\right)=-1.2$ for both cases. Thus even uncorrected metallicity effects in the spectral indices cannot reproduce the observed mass trends.

\subsection{Summary}

We propose a novel methodology to investigate galaxy properties through use of a combination of photometric and spectroscopic measurements. By using models of stellar population synthesis, we are able to recover a large array of physical properties of model galaxies using such a combination. In particular, we define a new quantity, SFA, which traces the instantaneous time derivative of the specific star formation rate of an individual galaxy by measuring the NUV $-i$ color time derivative, and which is also recovered by use of the aforementioned measurements.

The approach offers the following benefits:

1. Physical parameters are derived not by fitting but by a single matrix of linear coefficients.

2. The method makes no assumptions about star formation histories.

3. Moments of star formation history (the star formation rate and higher derivatives) can be derived nonparametrically.

4. The method works over all stellar masses with a single set of matrices; 
(a)

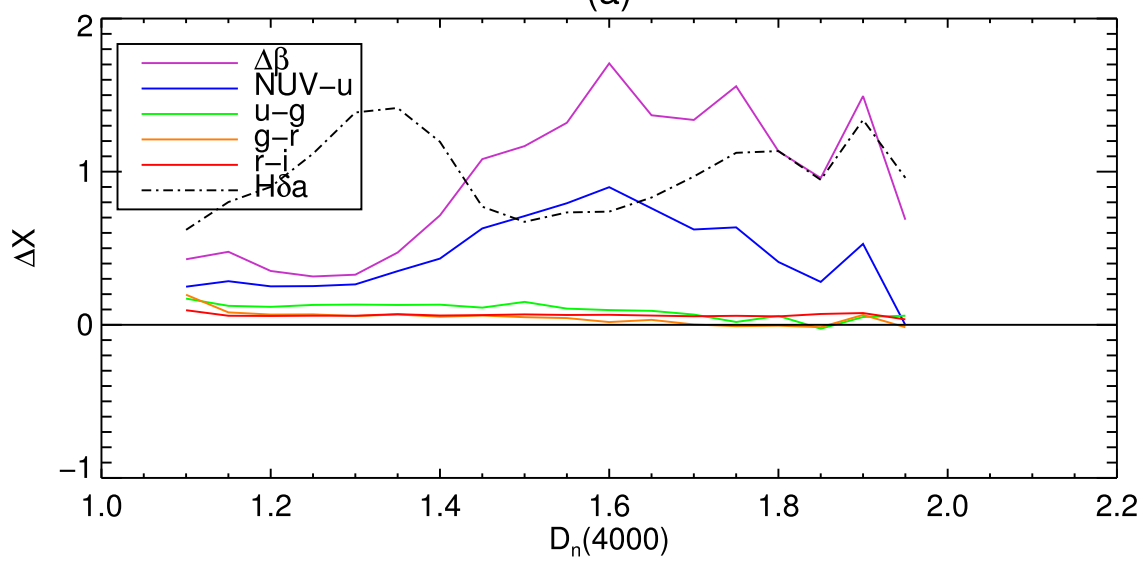

(b)

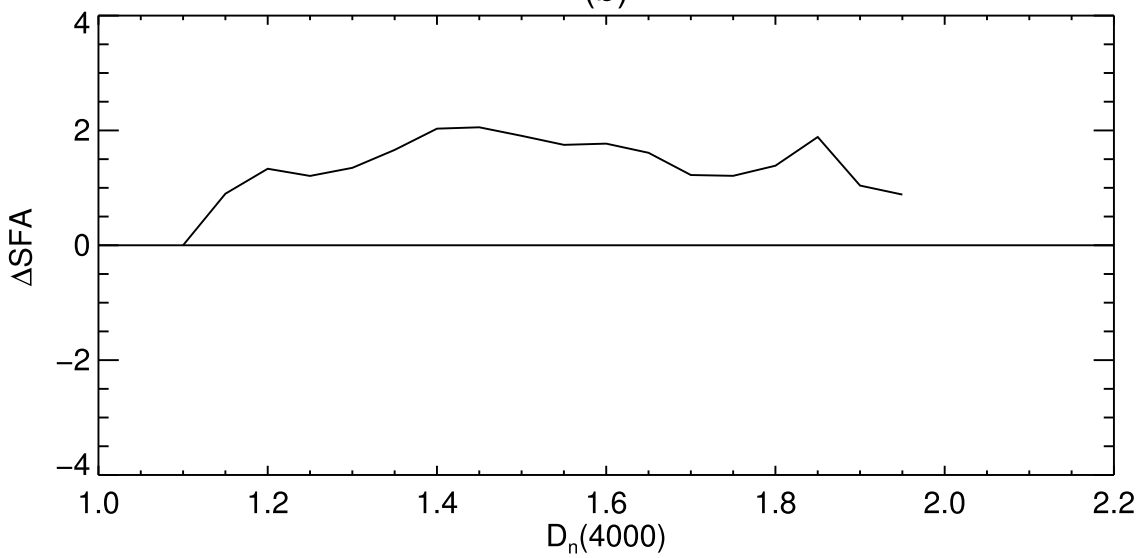

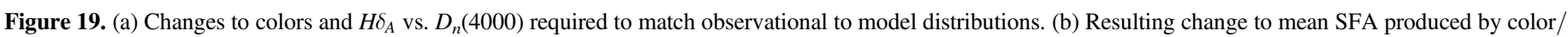
index changes vs. $D_{n}(4000)$.

Table 5

Color/Index Corrections

\begin{tabular}{lcrrrrr}
\hline \hline$D_{n}(4000)$ & $\beta$ & NUV $-u$ & $u-g$ & $g-r$ & NUV $-i$ & $H \delta_{a}$ \\
\hline 1.10 & 0.43 & 0.25 & 0.17 & 0.20 & 0.10 & 0.62 \\
1.15 & 0.48 & 0.28 & 0.12 & 0.08 & 0.06 & 0.80 \\
1.20 & 0.35 & 0.25 & 0.12 & 0.07 & 0.06 & 0.90 \\
1.25 & 0.32 & 0.25 & 0.13 & 0.07 & 0.06 & 1.12 \\
1.30 & 0.33 & 0.26 & 0.13 & 0.06 & 0.06 & 1.39 \\
1.35 & 0.47 & 0.35 & 0.13 & 0.07 & 0.07 & 1.42 \\
1.40 & 0.71 & 0.43 & 0.13 & 0.05 & 0.06 & 1.19 \\
1.45 & 1.08 & 0.63 & 0.11 & 0.06 & 0.06 & 0.77 \\
1.50 & 1.17 & 0.71 & 0.15 & 0.05 & 0.07 & 0.67 \\
1.55 & 1.32 & 0.79 & 0.11 & 0.04 & 0.06 & 0.73 \\
1.60 & 1.71 & 0.90 & 0.10 & 0.02 & 0.07 & 0.74 \\
1.65 & 1.37 & 0.76 & 0.09 & 0.03 & 0.06 & 0.83 \\
1.70 & 1.34 & 0.62 & 0.07 & 0.00 & 0.06 & 0.97 \\
1.75 & 1.56 & 0.64 & 0.02 & -0.01 & 0.06 & 1.12 \\
1.80 & 1.13 & 0.41 & 0.06 & -0.01 & 0.05 & 1.13 \\
1.85 & 0.96 & 0.28 & -0.03 & -0.02 & 0.07 & 0.95 \\
1.90 & 1.49 & 0.53 & 0.05 & 0.06 & 0.08 & 1.34 \\
1.95 & 0.69 & -0.00 & 0.06 & -0.02 & 0.04 & 0.96 \\
\hline
\end{tabular}

5. Degeneracies between the derived physical parameters and covariance are explicitly derived.

6. Error propagation is simple.

7. The influence of each observable on each derived physical parameter can be calculated and the resulting sensitivities provide useful context for error analysis and observation planning.
8. The method is easily generalized to incorporate new observables (e.g., morphological indices, other line indices, emission line fluxes, Sérsic indices, environmental parameters) and model-generated physical parameters (e.g., bulge-to-disk ratio, galaxy density).

9. The method is linear and therefore stacked spectra (within constant $D_{n}(4000)$ bins) can be used to derive average physical parameters. For example, galaxies can be stacked in bins (e.g., extinction-corrected color-magnitude bins), yielding an average physical parameter for the bin.

GALEX (Galaxy Evolution Explorer) is a NASA Small Explorer, launched in April 2003. We gratefully acknowledge NASA's support for construction, operation, and science analysis for the GALEX mission, developed in cooperation with the Centre National d'Etudes Spatiales of France and the Korean Ministry of Science and Technology. Behnam Darvish acknowledges financial support from NASA through the Astrophysics Data Analysis Program (ADAP), grant number NNX12AE20G. We thank the anonymous referee for valuable comments that strengthened the paper.

Facilities: GALEX, SDSS.

\section{Appendix A \\ Color and Spectral Index Correction and Impact on SFA}

We have adjusted model colors and spectral indices so that they are similar to those of the observed sample. We do this so 
(a)

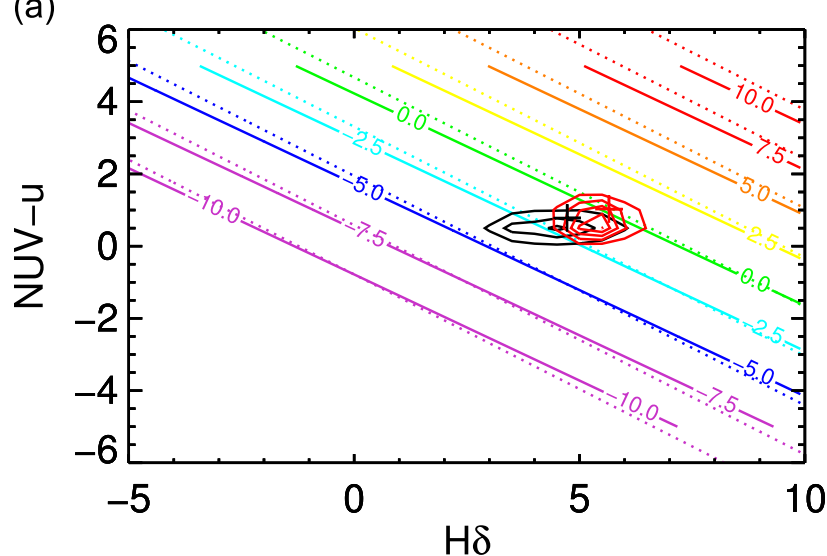

(b)

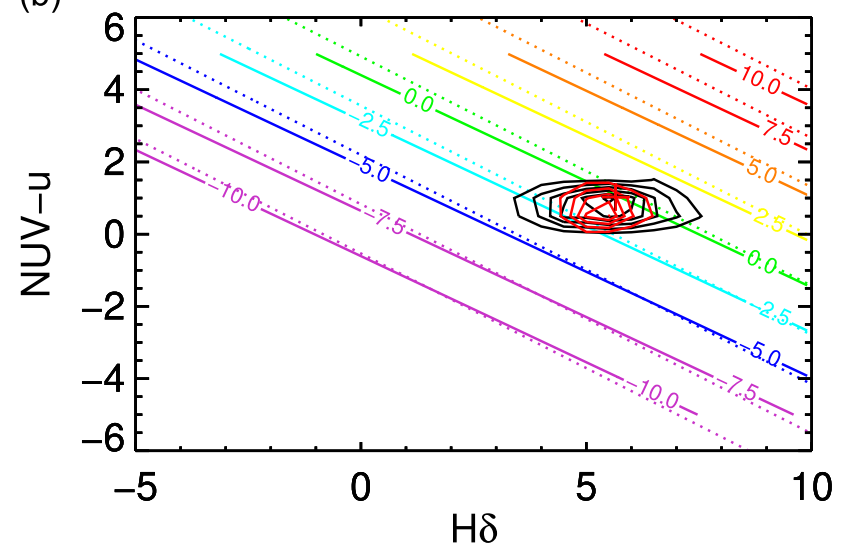

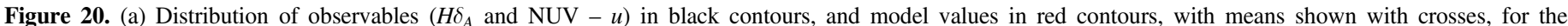

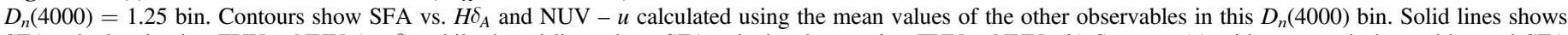

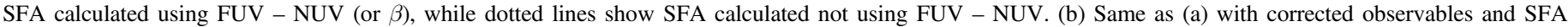
contours calculated using corrected mean observables.

(a)

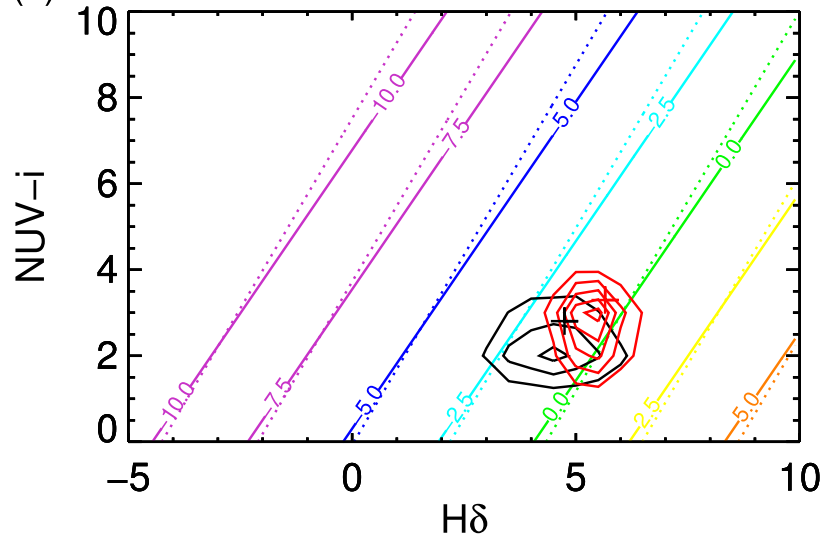

(b)

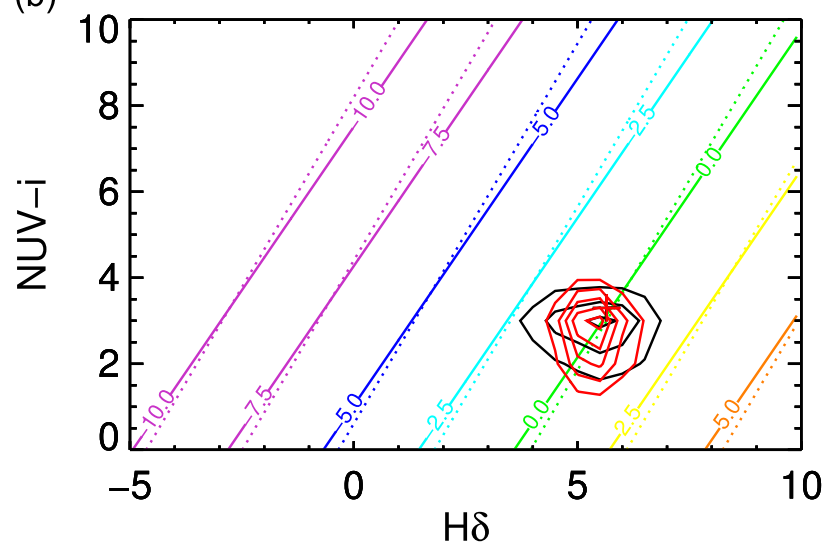

Figure 21. Same as Figure 20 for NUV $-i$ vs. $H \delta_{A}$.

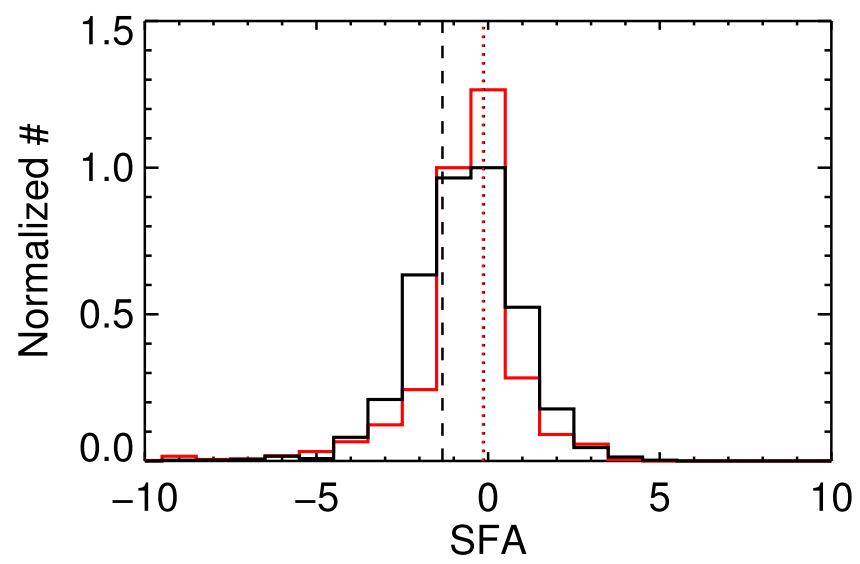

Figure 22. Histogram of derived SFA (with corrected observables, black) vs model SFA. Dotted lines show mean values (black: observable SFA, red: model SFA). The dashed line shows mean derived SFA for uncorrected observables. All are in the $D_{n}(4000)=1.25$ bin.

that the range of observational parameters used to extract the physical parameters is comparable to the model range. In order to make this comparison as representative as possible, we use a filtered sample of the model galaxies selected to be detected in
SDSS and GALEX NUV as a function of their redshift. In other words, the color-correction model sample is magnitude-limited in the same way as the observed sample. We note that the entire model sample was used to derive the regression coefficients, not the filtered sample.

For each $D_{n}(4000)$ bin, we compare the distribution of model and observed colors and indices, notably $H \delta_{A}$. We have tried using two methods: simple means and maximizing the crosscorrelation. These give results typically within $\sim 0.1$ in correction values. Our default is the mean method. For convenience we correct observables to model values, noting that this is equivalent to correcting model values to observable distributions (resulting in modified regression offsets), and permits the application of the published regression coefficients to other data sets.

We show a summary of the observable color/index changes in Figure 19(a) and tabulate them in Table 5. Note that the largest changes are to $\beta$ (the FUV - NUV slope parameter), NUV $-u$ (and correspondingly NUV $-i$ ), and $H \delta_{A}$. We also show in Figure 19(b) the impact on SFA. Over most of the range of $D_{n}(4000)$ there is an increase in SFA in the range of 0.7-2.0 mag $\mathrm{Gyr}^{-1}$, with a mean change of $\triangle \mathrm{SFA}=1.35$.

We give a few samples of the distributions in the next set of figures. In Figure 20, we show the distributions of 
(a)

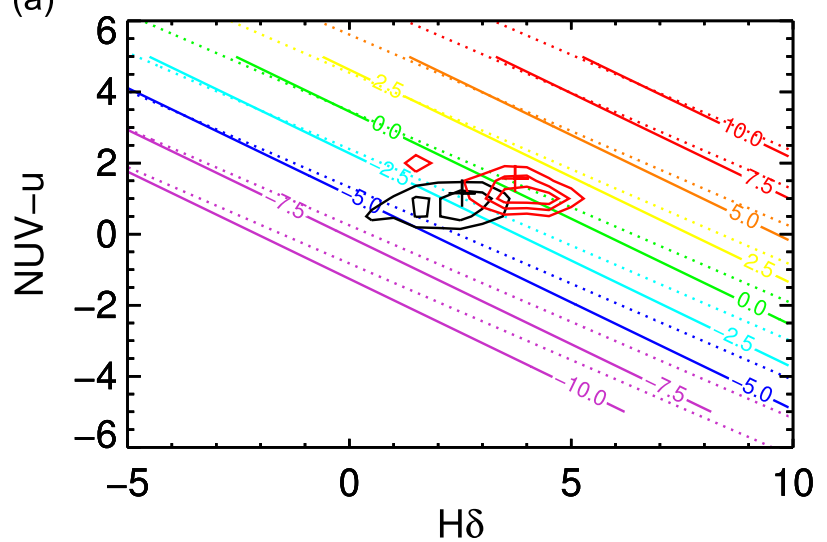

(b)

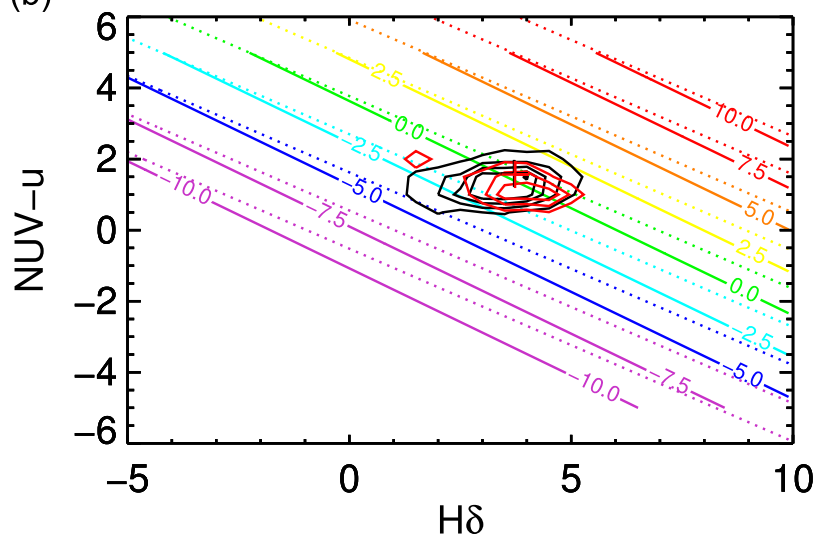

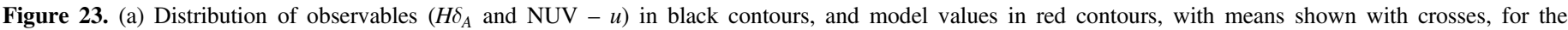

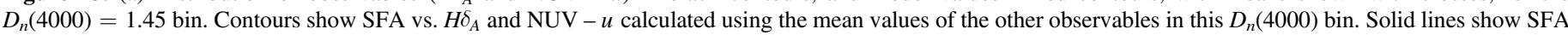

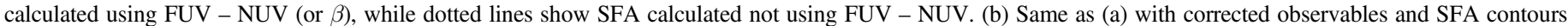
calculated using corrected mean observables.

(a)

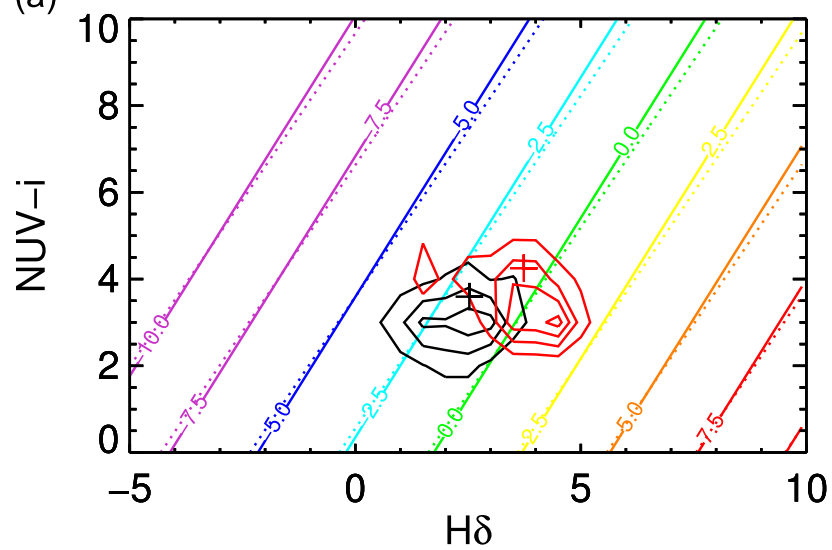

(b)

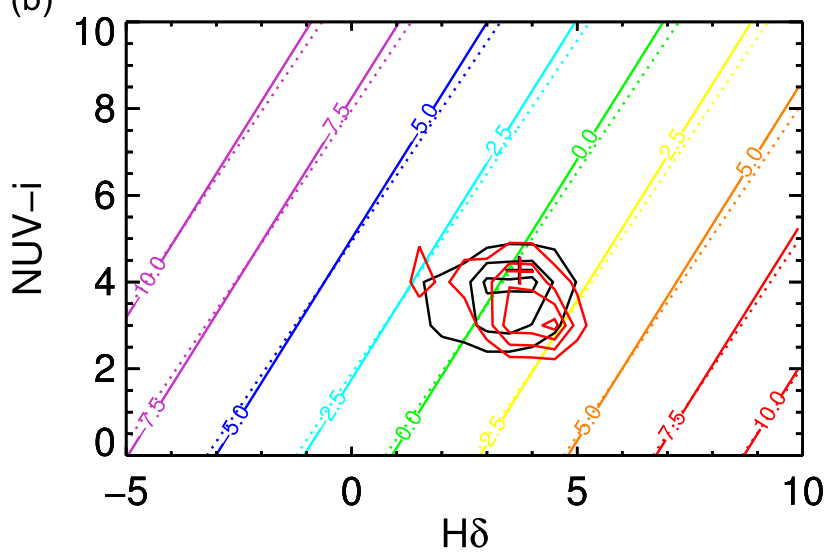

Figure 24. Same as Figure 23 for NUV $-i$ vs. $H \delta_{A}$.

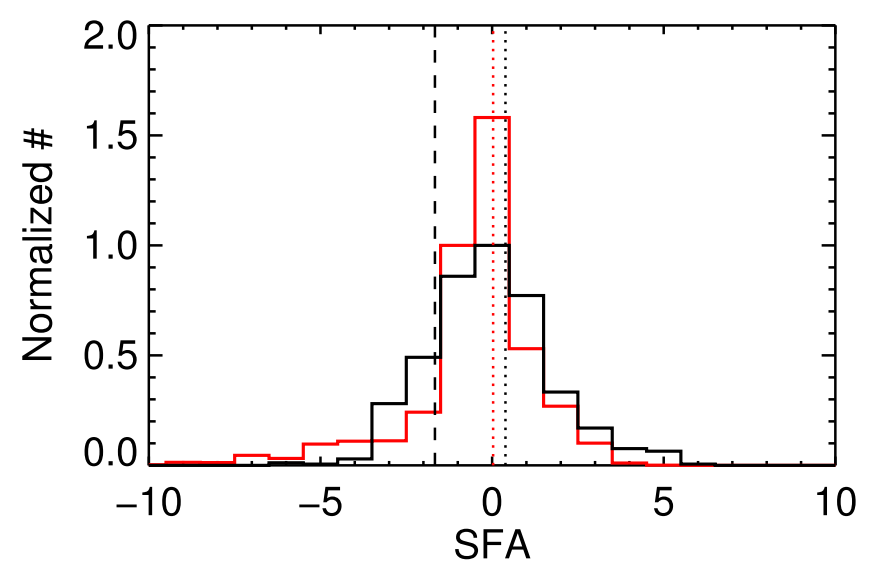

Figure 25. Histogram of derived SFA (with corrected observables, black) vs. model SFA. Dotted lines show mean values (black: observable SFA, red: model SFA). The dashed line shows mean derived SFA for uncorrected observables. All are in the $D_{n}(4000)=1.45$ bin.

observed NUV $-u$ and $H \delta_{A}$ for the $D_{n}(4000)=1.25$ bin. In Figure 20(a), we show the uncorrected observed values versus the model distribution. The plot also shows SFA contours using mean values for the other (observed) parameters, to show how variations in NUV $-u$ and $H \delta_{A}$ affect SFA. In Figure 20(b) we show the corrected observed values, model distribution, and SFA contours using the corrected mean observed values. See caption for further details. In Figure 21 we show the same information for NUV $-i$. We show the distributions of SFA in the $D_{n}(4000)=1.25$ bin in Figure 22. We repeat these figures for $D_{n}(4000)=1.45$ (Figures 23-25) and for $D_{n}(4000)=1.75$ (Figures 26-28). The figures showing the SFA distributions illustrate that the adjustments to observables bring the derived SFA into agreement with the model SFAs in their mean values. Without the corrections the two SFA distributions would be significantly discrepant. In general the spread in the derived SFA is similar to or greater than that in the model SFAs (Figures 22, 25, 28).

Finally in Figure 29 we show a version of Figure 12 with arrows added, indicating how the observable corrections and associated changes in SFA impact the flux diagram. There is a modest impact, typically moving the quench/burst point about 0.1 dex down in the quench direction in $\log (\mathrm{sSFR})$.

We note as further evidence of the validity of this approach that the quenching rate derived in Table 4 and Figure 13 is consistent with the results of Martin et al. (2007), which were 
(a)

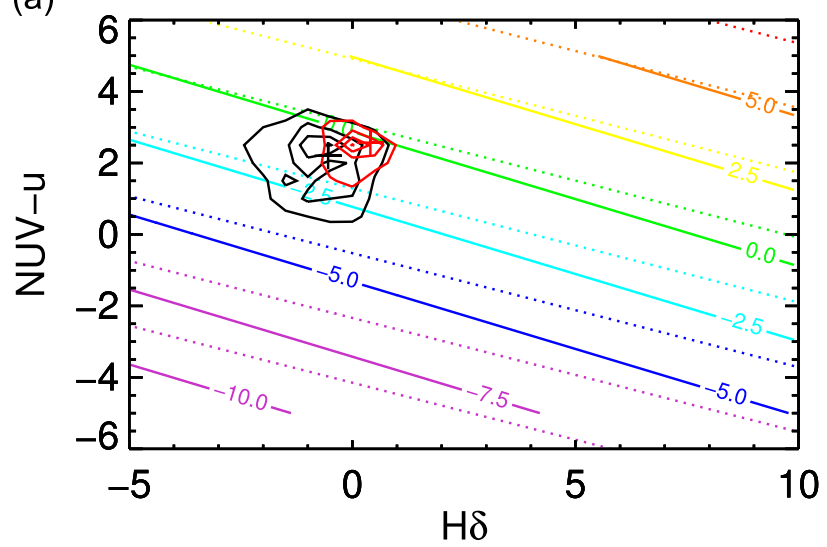

(b)

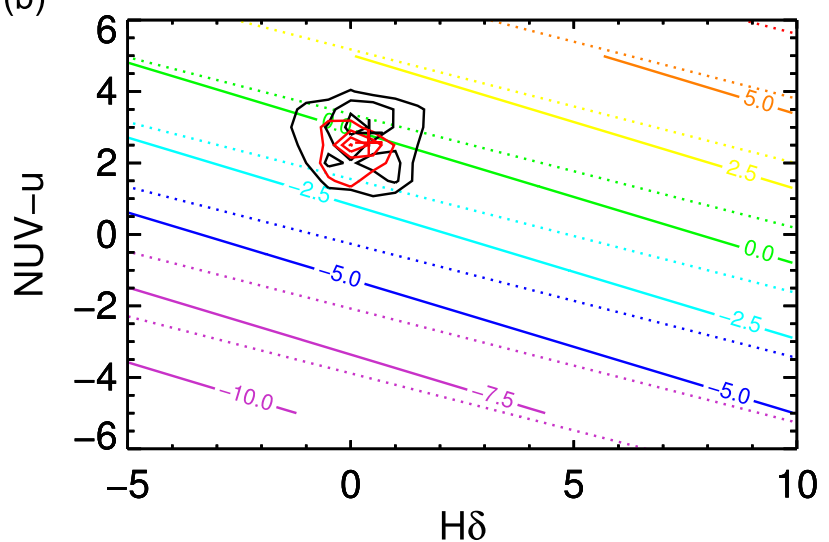

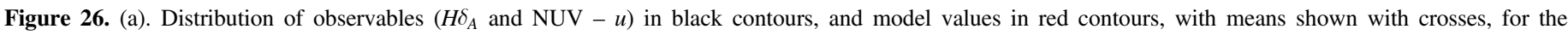

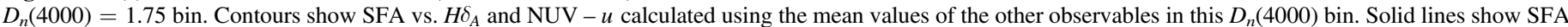

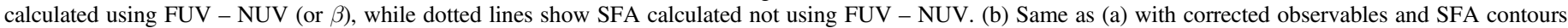
calculated using corrected mean observables.

(a)

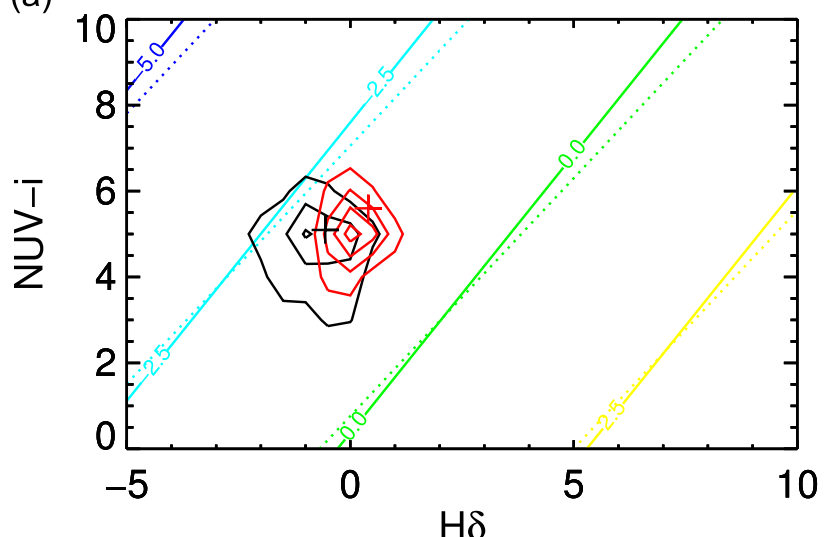

(b)

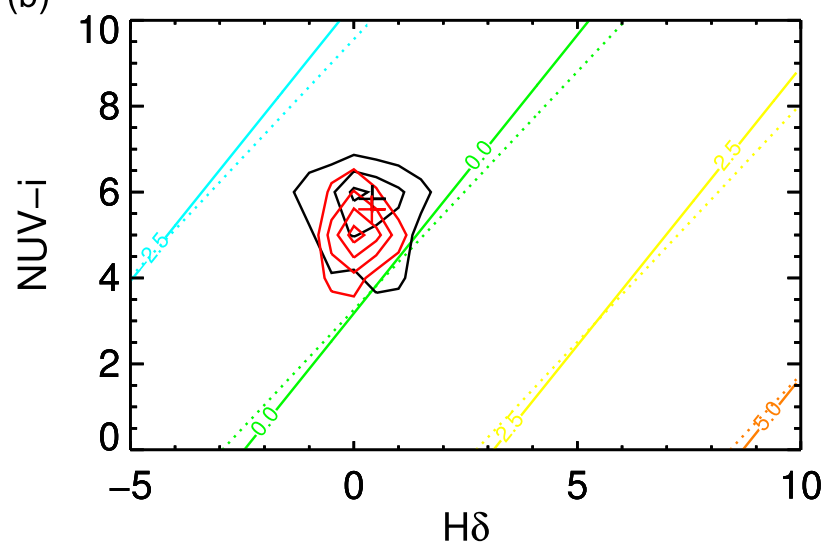

Figure 27. Same as Figure 26 for NUV $-i$ vs. $H \delta_{A}$.

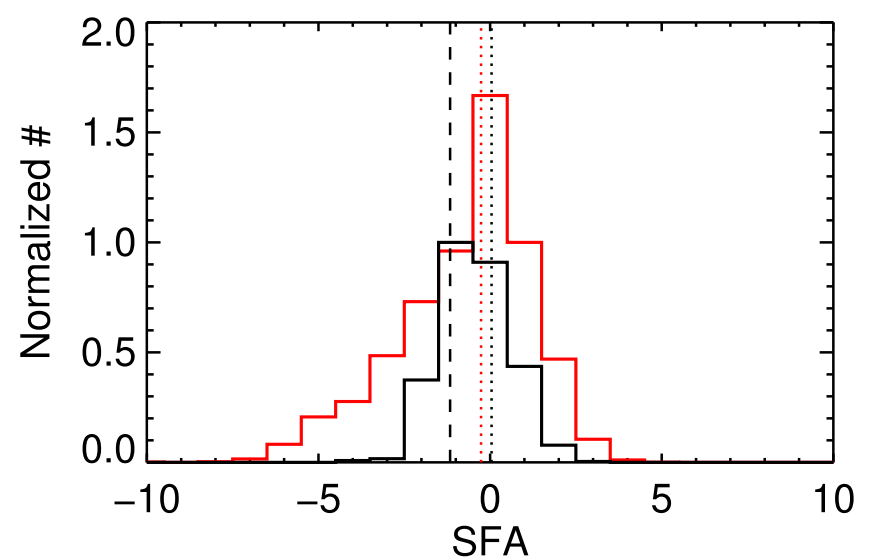

Figure 28. Histogram of derived SFA (with corrected observables, black) vs. model SFA. Dotted lines show mean values (black: observable SFA, red: model SFA). The dashed line shows mean derived SFA for uncorrected observables. All are in the $D_{n}(4000)=1.75$ bin.

obtained using an independent method. Both of these results are quantitatively consistent with the observed evolution in the galaxy main sequence and red sequences. Without reconciling the model and observation distributions, there would be a very significant discrepancy between the derived quenching mass flux and the evolution of the main and red sequences.

\section{Appendix B \\ Comparison to Semianalytic Model Trends}

We have repeated the analysis of Section 4.2 for the model galaxies used to generate the fitting coefficients. We choose galaxies in the redshift range $0<z<0.3$ using the magnitude-limited subset discussed above. The mean SFA over this diagram is given in Figure 30(a) (the equivalent of Figure 11). We also show the impact of fitting error on this diagram in Figure 30(b). This shows that biases introduced by fitting SFA are typically 0.0-0.5. Correcting for these small biases on this diagram would slightly amplify the observed trends. In Figure 31 we show the flux diagram that is the equivalent of Figure 12. The trends with sSFR (SFA decreasing) and with mass (SFA decreasing with increasing mass in the green valley) are similar, but the amplitudes are smaller. When we perform the equivalent of the calculation of Table 4, we find a mass flux (over the same mass range) of $\dot{\rho}_{\mathrm{BR}}=3.3 \times 10^{-4} M_{\odot} \mathrm{yr}^{-1} \mathrm{Mpc}^{-3}$, a factor of $\sim 100$ lower than our observed mass flux and that inferred from the evolution of the blue and red galaxy luminosity functions. 


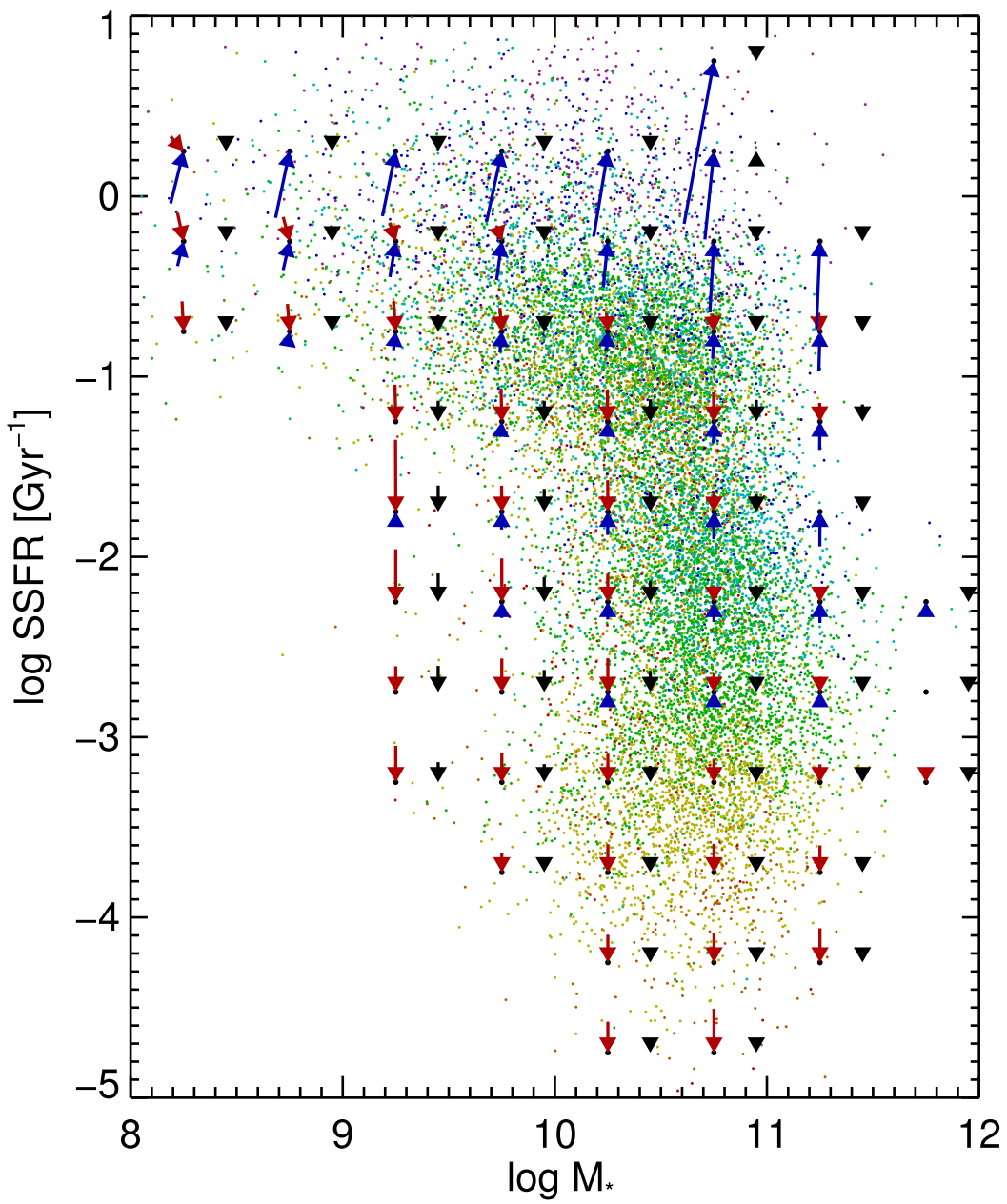

Figure 29. Star formation acceleration (SFA) plotted as a flux vector on the sSFR vs. $M_{*}$ diagram for SDSS galaxies. Same as Figure 12. Added black arrows show change in quench/burst mean point produced by change in SFA from correction of the observable distribution.
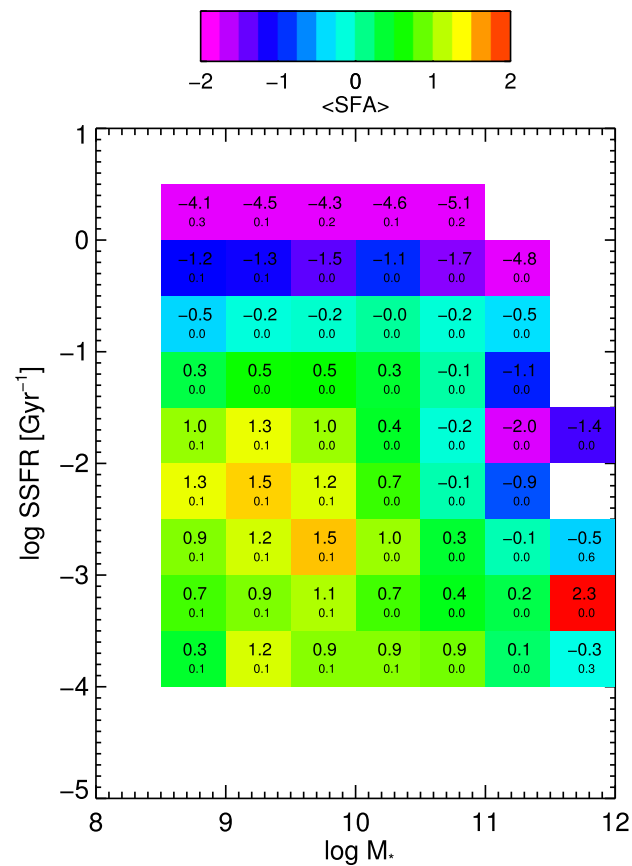
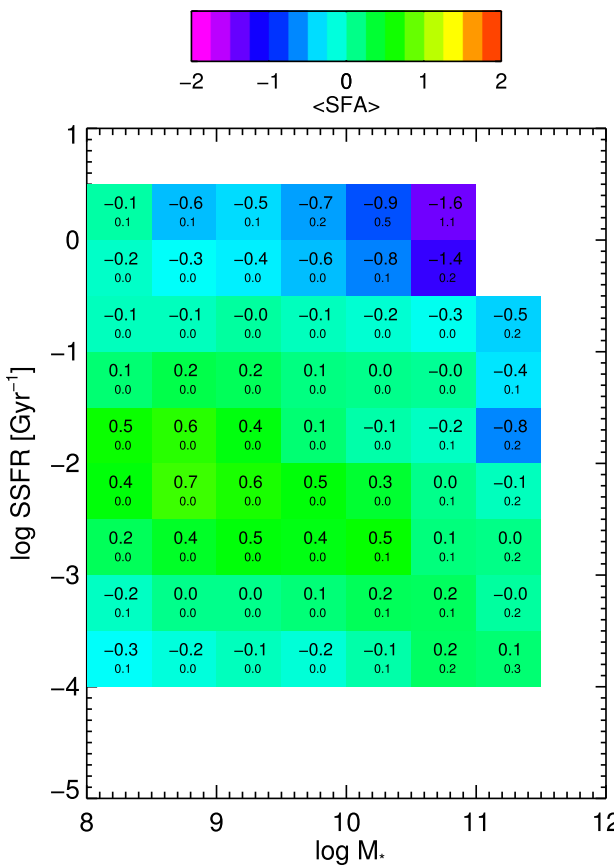

Figure 30. (a) Mean star formation acceleration (SFA) indicated by color on the $\log$ (sSFR) vs. $\log M_{*}$ diagram for model galaxies. Compare to Figure 11 . (b) Difference between model SFA and regression fit SFA ( $\left.(\mathrm{SFA}\rangle-\left\langle\mathrm{SFA}_{\mathrm{fit}}\right\rangle\right)$ averaged in each $\log (\mathrm{sSFR})-\log M_{*}$ bin. Maximum differences are typically $0.0-0.5$ over most of the diagram. Correcting for these biases would slightly increase the mass trends discussed in the text. 


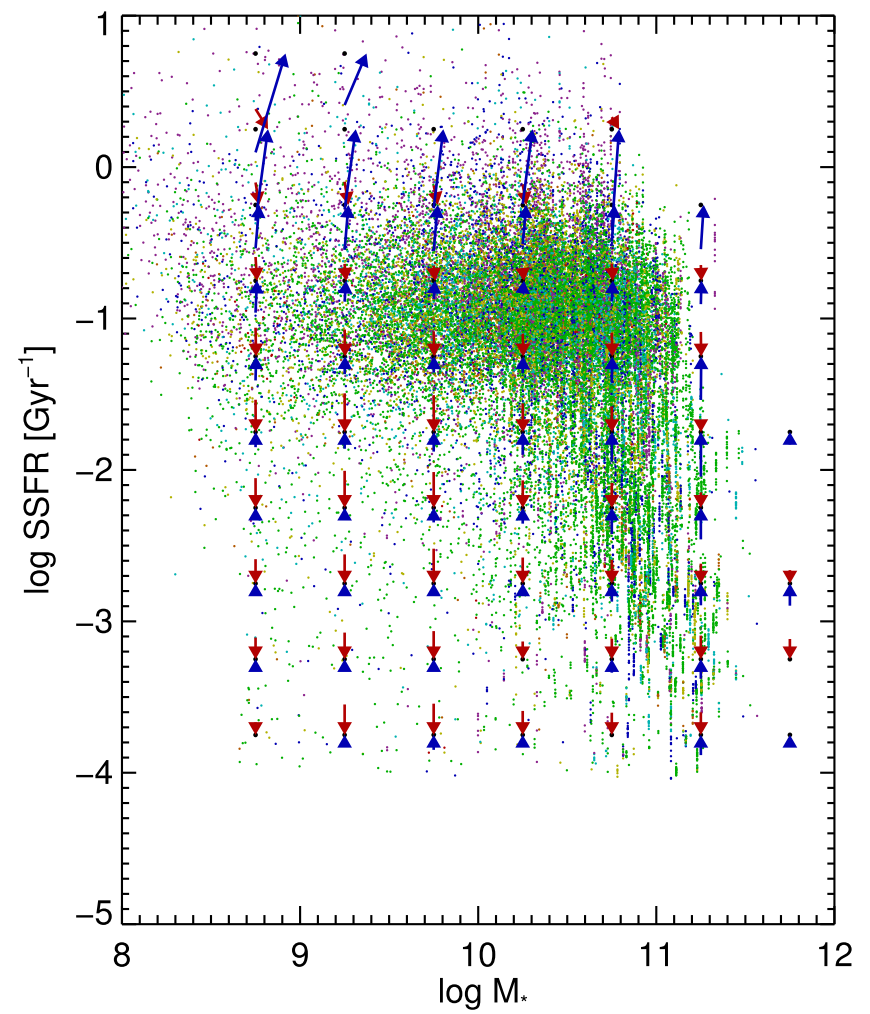

Figure 31. Star formation acceleration (SFA) plotted as a flux vector on the sSFR vs. $M_{*}$ diagram for model galaxies. Compare to Figure 12.

\section{References}

Aird, J., Coil, A. L., Moustakas, J., et al. 2012, ApJ, 746, 90 Baldry, I. K., Glazebrook, K., Brinkmann, J., et al. 2004, ApJ, 600, 681 Balogh, M. L., Baldry, I. K., Nichol, R., et al. 2004, ApJ, 615, 101 Bell, E. F., Wolf, C., Meisenheimer, K., et al. 2004, ApJ, 609, 752 Bigiel, F., Leroy, A., Walter, F., et al. 2008, AJ, 136, 2846 Blanton, M. 2006, arXiv:astro-ph/0512127

Blanton, M., Eisenstein, D., Hogg, D. W., Schlegel, D. J., \& Brinkmann, J. 2005, ApJ, 629, 143

Bruzual, G., \& Charlot, S. 2003, MNRAS, 344, 1000

Calzetti, D., Kinney, A. L., \& Storchi-Bergmann, T. 1994, ApJ, 429, 582

Calzetti, D., Armus, L., Bohlin, R. C., et al. 2000, ApJ, 533, 682

Cardelli, J. A., Clayton, G. C., \& Mathis, J. S. 1989, ApJ, 345, 245

Cheung, E., Faber, S. M., Koo, D. C., et al. 2012, ApJ, 760, 131

Coil, A., Weiner, B. J., Holz, D. E., et al. 2011, ApJ, 743, 46
Croton, D., Springel, V., White, S. D. M., et al. 2006, MNRAS, 365, 11 Darvish, B., Mobasher, B., Sobral, D., et al. 2016, ApJ, 825, 113

De Lucia, G. 2006, MNRAS, 366, 499

Di Matteo, T., Springel, V., \& Hernquist, L. 2005, Natur, 433, 604

Dressler, A. 1980, ApJ, 236, 351

Dubois, Y., Gavazzi, R., Peirani, S., \& Silk, J. 2013, MNRAS, 433, 3297

Faber, S. M., Willmer, C. N. A., Wolf, C., et al. 2007, ApJ, 665, 265

Fang, J., Faber, S. M., Salim, S., Graves, G. J., \& Rich, R. M. 2012, ApJ, 761, 23

Gaibler, V., Khochfar, S., Krause, M., \& Silk, J. 2012, MNRAS, 425, 438

Gonçalves, T. S., Martin, D. C., Menéndez-Delmestre, K., Wyder, T. K., \& Koekemoer, A. 2012, ApJ, 759, 67

Ilbert, O., McCracken, H. J., Le Fèvre, O., et al. 2013, A\&A, 556, 55

Johnson, B. D., Schiminovich, D., Seibert, M., et al. 2007a, ApJS, 173, 377

Johnson, B. D., Schiminovich, D., Seibert, M., et al. 2007b, ApJS, 173, 392

Kauffman, G., Heckman, T. M., White, S. D. M., et al. 2003, MNRAS, 341 , 33

Kennicutt, R. C., Jr. 1998, ApJ, 498, 541

King, A. 2005, ApJL, 635, 121

Maiolino, R., Gallerani, S., Neri, R., et al. 2012, MNRAS, 425, L66

Mancini, C., Renzini, A., Daddi, E., et al. 2015, MNRAS, 450, 763

Martin, D. C., Fanson, J., Schiminovich, D., et al. 2005, ApJL, 619, L1

Martin, D. C., Wyder, T. K., Schiminovich, D., et al. 2007, ApJS, 173, 342

Nandra, K. 2007, ApJL, 660, 11

Oke, J. B., \& Gunn, J. E. 1983, ApJ, 266, 713

Olsen, K. P., Rasmussen, J., Toft, S., \& Zirm, A. W. 2013, ApJ, 764, 4

Peng, Y., Lilly, S. J., Kovač, K., et al. 2010, ApJ, 721, 193

Peng, Y., Maiolino, R., \& Cochrane, R. 2015, Natur, 521, 192

Rampazzo, R., Marino, A., Tantalo, R., et al. 2007, MNRAS, 381, 245

Rovilos, E., Comastri, A., Gilli, R., et al. 2012, A\&A, 546, 58

Salim, S., Charlot, S., Rich, R. M., et al. 2005, ApJL, 619, 39

Salim, S., Rich, R. M., Charlot, S., et al. 2007, ApJS, 173, 267

Salim, S., Fang, J. J., Rich, R. M., Faber, S. M., \& Thilker, D. A. 2012, ApJ, 755,105

Salpeter, E. E. 1955, ApJ, 121, 161

Schawinski, K., Lintott, C. J., Thomas, D., et al. 2009, ApJ, 690, 1672

Seibert, M., Martin, D. C., Heckman, T. M., et al. 2005, ApJL, 619, L55

Shimizu, T. T., Mushotzky, R. F., Meléndez, M., Koss, M., \& Rosario, D. J. 2015, MNRAS, 452, 1841

Springel, V., DiMatteo, T., \& Hernquist, L. 2005, ApJL, 620, L79

Springel, V., White, S. D. M., Jenkins, A., et al. 2005, Natur, 435, 629

Thilker, D., Bianchi, L., Schiminovich, D., et al. 2010, ApJL, 714, 171

Thomas, D., Maraston, C., Bender, R., \& Mendes de Oliveira, C. 2005, ApJ, 621,673

Thomas, D., Maraston, C., Schawinski, K., Sarzi, M., \& Silk, J. 2010, MNRAS, 404, 1775

Tremonti, C. A., Heckman, T. M., Kauffmann, G., et al. 2004, ApJ, 613 898

Willmer, C. N. A., Faber, S. M., Koo, D. C., et al. 2006, ApJ, 647, 853

Wyder, T. K., Martin, D. C., Barlow, T. A., et al. 2009, ApJ, 696, 1834

Wyder, T., Martin, D. C., Schiminovich, D., et al. 2007, ApJS, 173, 293

Zehavi, I., Blanton, M. R., Frieman, J. A., et al. 2005, ApJ, 571, 172 